Reservoir Rëlease Patterns fór Hydropower Operations at the Aspinall Unit on the Gunnison River, Colorado

Environmental Assessment Bívision Argonne National Laboratory

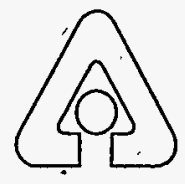

Operated by The University of Chicago, under Contract W-31-109-Eng-38, for the United States Department of Energy 


\section{Argonine National Laboratory}

Argonne National Laboratory, with facilities in the states of Illinois and ldaho, is owned by the United States Government, and operated by the University of Chicago under the provisions of a contract with the Department of Energy:

This technical memo is a product of Argonne's Environimental Assessment Division (EAD). For information on the division's scientific and engineering activities, contact:

Director, Envirónmental Assessment Division

Argonne National Laboratory

Argonne, Illinois 60439-4815

Telephone (708) 252-3107

Presented in this technical memo are preliminary results of ongoing work or work that is more limited in scope and depth than that described in formal reports issued by the EAD.

Publishing support services were provided by Àrgonne's Information and Publishing Division.

\section{Disclaimer}

This report was prepared as an account of work sponsored by an agency of the United States Government. Neither the United States Government nor any agency thereof, nor any of their employees, makes any wananty, express or implied, or assumes any legal liability or responsibility for the accuracy, completeness, or usefulness of any information, apparatus, product; or process disclosed, or represents that its use would not infringe privately owned rights. Reference herein to any specific commercial product, process, or service by trade name, trademark, manufacturer, or otherwise; does not necessarily constitute or imply it's endorsement,recommendation; or favoring by the United States Government or any. agency thereof. The views and opinions of authors expressed herein do not necessarily state or reflect those of the United States.Govemment or any agency thereof.

Reproduced directly from the best available copy. Available to DOE and DOE contractors from the Office of Scientific and Technical Information, P.O.'Box'62, Cak Ridge, TN 37831; prices available from (615) $576-8401$.

Available to the public from the National Technical information Service, U.S. Department of Commerce, 5285 Port Royal Road, Springfield, VA 22161. 


\section{DISCLAIMER}

Portions of this document may be illegible in electronic image products. Images are produced from the best available original document. 


\section{Reservoir Release Patterns for Hydropower Operations at the Aspinall Unit on the Gunnison River, Colorado}

by S.C.L. Yin, J.J. McCoy, ${ }^{\star}$ and J. Sedlacek ${ }^{\star}$

Environmental Assessment Division,

Argonne National Laboratory, 9700 South Cass Avenue, Argonne, Illinois 60439

May 1995

Work sponsored by United States Department of Energy, Western Area Power Administration

"McCoy is affiliated with Western Area Power Administration, Salt Lake City, Utah, and Sedlacek with PLM Technologies, Evergreen, Colorado. 
This report is printed on recycled paper. 


\section{FOREWORD}

This report is one of a series of technical memorandums prepared to support an environmental impact statement (EIS) on power marketing prepared by Argonne National Laboratory for the U.S. Department of Energy's Western Area Power Administration (Western). Western markets electricity produced at hydroelectric facilities operated by the Bureau of Reclamation. The facilities are known collectively as the Salt Lake City Area Integrated Projects (SLCA/IP) and include dams equipped for power generation on the Colorado, Green, Gunnison, and Rio Grande rivers and on Plateau Creek in the states of Arizona, Colorado, New Mexico, Utah, and Wyoming.

Western proposes to establish a level of commitment (sales) of long-term firm electrical capacity and energy from the SLCA/IP hydroelectric power plants; the impacts of this proposed action are evaluated in the EIS. Of the SLCA/IP facilities, only the Glen Canyon Dam, Flaming Gorge Dam, and Aspinall Unit (which includes Blue Mesa, Morrow Point, and Crystal dams) are influenced by Western's power scheduling and transmission decisions. For this reason, the impacts of hydropower operations at these three facilities were examined in the EIS.

The technical memorandums present detailed findings of studies conducted by Argonne National Laboratory specifically for the EIS. These studies are summarized in the EIS, and the results were used to assess environmental impacts related to alternative commitment levels. Technical memorandums were prepared on a number of socioeconomic and natural resource topics. Staff members of Argonne National Laboratory's Decision and Information Sciences Division and Environmental Assessment Division prepared these technical memorandums and the EIS as part of a joint effort managed by the Environmental Assessment Division. 


\section{CONTENTS}

FOREWORD $\ldots \ldots \ldots \ldots \ldots \ldots \ldots \ldots \ldots \ldots \ldots \ldots \ldots \ldots \ldots \ldots \ldots \ldots \ldots \ldots \ldots \ldots$ iii

ABSTRACT $\ldots \ldots \ldots \ldots \ldots \ldots \ldots \ldots \ldots \ldots \ldots \ldots \ldots \ldots \ldots \ldots \ldots \ldots \ldots$

1 INTRODUCTION $\ldots \ldots \ldots \ldots \ldots \ldots \ldots \ldots \ldots \ldots \ldots \ldots \ldots \ldots \ldots \ldots \ldots \ldots$

2 DEVELOPMENT OF RESERVOIR RELEASE PATTERNS $\ldots \ldots \ldots \ldots \ldots \ldots 2$

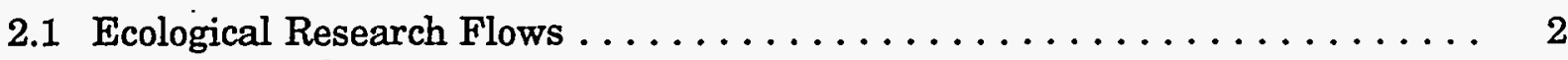

2.2 Reservoir Release Patterns . . . . . . . . . . . . . . . . . 3

2.2.1 Seasonally Adjusted Steady Flows $\ldots \ldots \ldots \ldots \ldots \ldots \ldots \ldots, 4$

2.2.2 Seasonally Adjusted High Fluctuating Flows $\ldots \ldots \ldots \ldots \ldots \ldots$

3 RESERVOIR SURFACE FLUCTUATIONS $\ldots \ldots \ldots \ldots \ldots \ldots \ldots \ldots$

4 WATER SURFACE ELEVATIONS BELOW MORROW POINT DAM . . . . . . . 5

5 CONCLUSIONS ................................. 6

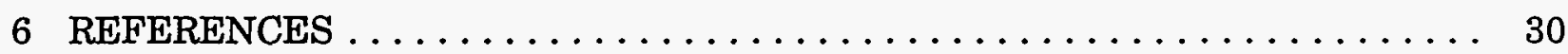

\section{FIGURES}

1 Locations of Flaming Gorge Dam, Glen Canyon Dam, and the Aspinall Unit . . . . . . . . . . . . . . . . . .

2 Locations of Blue Mesa, Morrow Point, and Crystal Reservoirs on the Gunnison River, Colorado ................. 8

3 Research and Historical Releases at Crystal Reservoir ............. 9

4 Water Surface Elevations below Morrow Point Dam ................ 10

\section{TABLES}

1 Monthly Releases from Crystal Reservoir for Moderate, Dry, and Wet Years

2 Reservoir Operations for the Seasonally Adjusted Steady Flow Scenario at the Aspinall Unit during a Moderate Year

3 Reservoir Operations for the Seasonally Adjusted Steady Flow Scenario at the Aspinall Unit during a Dry Year 


\section{TABLES (Cont.)}

4 Reservoir Operations for the Seasonally Adjusted Steady Flow Scenario at the Aspinall Unit during a Wet Year $\ldots \ldots \ldots \ldots \ldots$

5 Bureau of Reclamation Coefficient Tables Used to Calculate

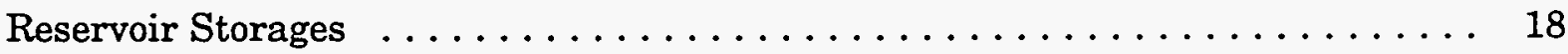

6 Daily Reservoir Operations at the Aspinall Unit, October $1982 \ldots \ldots$. . . . . . 19

7 Adjusted Daily Releases at Crystal Reservoir, April

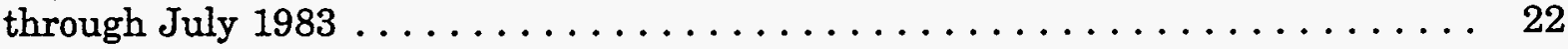

8 Maximum Allowable Fluctuations for Reservoir Releases

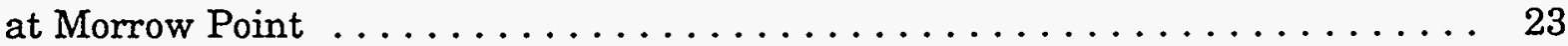

9 Daily Release Patterns for Blue Mesa Reservoir . . . . . . . . . . . . . . . . . 24

10 Daily Release Patterns for Morrow Point Reservoir . . . . . . . . . . . . . . 25

11 Maximum Daily Reservoir Surface Fluctuations under the Seasonally Adjusted High Fluctuating Flow Scenario during a Moderate Year $\ldots \ldots \ldots \ldots \ldots \ldots \ldots \ldots$

12 Maximum and Minimum Reservoir Surface Elevations on the First Day of the Month under the Seasonally Adjusted High Fluctuating Flow Scenario at the Aspinall Unit during a Moderate Year $\ldots \ldots \ldots \ldots \ldots \ldots \ldots \ldots \ldots \ldots \ldots \ldots \ldots \ldots \ldots \ldots$ 


\title{
RESERVOIR RELEASE PATTERNS FOR HYDROPOWER OPERATIONS AT THE ASPINALL UNIT ON THE GUNNISON RIVER, COLORADO
}

by

\author{
S.C.L. Yin, J.J. McCoy, and J. Sedlacek
}

\begin{abstract}
This report presents the development of reservoir release patterns for the Aspinall Unit, which includes Blue Mesa, Morrow Point, and Crystal Reservoirs on the Gunnison River in Colorado. Release patterns were assessed for two hydropower operational scenarios - seasonally adjusted steady flows and seasonally adjusted high fluctuating flows - and three representative hydrologic years - moderate (1987), dry (1989), and wet (1983). The release patterns for the operational scenarios were developed with the aid of monthly, daily, and hourly reservoir operational models, which simulate the linked operation of the three Aspinall Unit reservoirs. Also presented are reservoir fluctuations and downstream water surface elevations corresponding to the reservoir release patterns. Both of the hydropower operational scenarios evaluated are based on the ecological research flows proposed by the U.S. Fish and Wildlife Service for the Aspinall Unit. The first operational scenario allows only seasonally adjusted steady flows (no hourly fluctuations at any dam within one day), whereas the second scenario permits high fluctuating flows from Blue Mesa and Morrow Point Reservoirs during certain times of the year. Crystal Reservoir would release a steady flow within each day under both operational scenarios.
\end{abstract}

\section{INTRODUCTION}

The Salt Lake City Office of the Western Area Power Administration (Western) markets electricity produced at hydroelectric facilities operated by the Bureau of Reclamation on the Upper Colorado River Basin (the subbasin of the Colorado River basin upstream of Glen Canyon Dam). The facilities are known collectively as the Salt Lake City Area Integrated Projects. Hydropower operations at three of these facilities are affected by Western power marketing activities: Glen Canyon Dam on the Colorado River, Flaming Gorge Dam on the Green River, and the Aspinall Unit on the Gunnison River (Figure 1*). This report presents the development of the Aspinall Unit release patterns and the resulting reservoir surface fluctuations and downstream water surface elevations for two hydropower

\footnotetext{
*For readability, all figures and tables are placed in sequence following Section 5 of this report.
} 
operational scenarios - seasonally adjusted steady flows and seasonally adjusted high fluctuating flows. The results presented here were used for assessing potential impacts of the hydropower operational scenarios on ecological, cultural, recreational, and other natural resources in the vicinity of the Aspinall Unit. The results of these assessments are presented in the environmental impact statement (EIS).

\section{DEVELOPMENT OF RESERVOIR RELEASE PATTERNS}

The Aspinall Unit on the Gunnison River in Colorado consists of Blue Mesa Reservoir, Morrow Point Reservoir, and Crystal Reservoir in downstream order (Figure 2). On the basis of streamflow records, water years 1987, 1989, and 1983 were selected as representative moderate, $\mathrm{dry}$, and wet years, respectively. (A water year begins on October 1 of the preceding calendar year and ends on September 31 of the current calendar year, e.g., water year 1987 begins on October 1, 1986, and ends on September 30,1987.) Reservoir release patterns were developed for these three representative hydrologic years.

The two hydropower operational scenarios evaluated in this report - seasonally adjusted steady flows and seasonally adjusted high fluctuating flows - are both based on the ecological research flows proposed by the U.S. Fish and Wildlife Service for the Aspinall Unit (Harris 1992). The first scenario allows only seasonally adjusted steady flows (no hourly fluctuation within a day), whereas the second scenario permits seasonally adjusted high fluctuating flows from Blue Mesa and Morrow Point Reservoirs within the requirements of research flows. Crystal Reservoir would release only seasonally adjusted steady flows within each day under both operational scenarios.

\subsection{ECOLOGICAL RESEARCH FLOWS}

The monthly releases from Crystal Reservoir for the representative water years are shown in Table 1. The releases for 1987 and 1989 are the flows below Crystal Dam that were proposed for ecological research by the U.S. Fish and Wildlife Service for representative moderate and dry water years, respectively; also proposed were research flows for a wet year, 1985 (Harris 1992). Because the representative wet year for this analysis is 1983 rather than 1985, monthly research flows for 1983 were developed on the basis of the 1985 research flow pattern. The research flows below Crystal Dam are presented graphically in Figure 3. For comparison, actual historical releases from Crystal Reservoir for 1987, 1989, and 1983 are also shown in Figure 3.

Release patterns from the two upstream reservoirs - Blue Mesa and Morrow Point - were developed on the basis of the Crystal Dam research flows. The feasibility of these releases from the three reservoirs was confirmed by monthly reservoir operational models for the three representative years based on historical inflows to Blue Mesa and side inflows to Morrow Point and Crystal Reservoirs. Initial reservoir storages were also based on historical values, except for the 1987 initial storage for Blue Mesa. An initial storage of 
660,000 acre-feet, lower than the historical 742,000 acre-feet, was necessary for the proposed Crystal research flows to be feasible. Operations of reservoirs upstream from Blue Mesa could be modified to provide such a lower initial storage. Tables 2, 3, and 4 show reservoir operations based on the research flows for 1987, 1989, and 1983, respectively. The reservoir surface elevations shown in the tables were computed from the storage-elevation relationships presented in Table 5.

\subsection{RESERVOIR RELEASE PATTERNS}

Reservoir release patterns for all three reservoirs under either of the two operational scenarios might be restricted by the operating constraints for Crystal Reservoir, which could have large surface fluctuations due to its relatively small storage capacity. The feasibility of the operational scenarios on both daily and hourly bases was assessed by developing daily and hourly reservoir operational models to simulate the linked operations of the three Aspinall Unit reservoirs. The reservoir operation was simulated by accounting daily inflows (from upstream and side inflows between two dams) and outflows (reservoir release and evaporation loss) and, subsequently, determining end-of-day reservoir storage and corresponding reservoir surface elevation. The water travel time between a dam and the head of the next downstream reservoir was ignored because of the short distances, which, even under low reservoir conditions, are less than $1 \mathrm{mi}$.

The daily operational models are illustrated in Table 6, which shows the spreadsheet setup and the first month of the numerical simulation for each reservoir operation. The hourly operational model is similar, except that the time interval is one hour. The daily operational model was validated by simulating historical reservoir operations for 1987, 1989, and 1983, using recorded daily reservoir inflows and releases and estimated evaporation losses. Calculated end-of-month water surface elevations for all three reservoirs were found to deviate generally less than $1 \mathrm{ft}$ from those reported by the Bureau of Reclamation. The reservoir operational models were developed with the spreadsheet program Quattro Pro run on an IBM-compatible personal computer.

The releases from Crystal Reservoir, including hourly release fluctuations, are sometimes limited to enhance the stability of the reservoir side-slope. In wet seasons (April through July), the reservoir surface elevation is not allowed to change by more than $0.5 \mathrm{ft}$ in any 24-hour period if the elevation is lower than 6,748 ft. If the elevation is at or above $6,748 \mathrm{ft}$, the elevation is not allowed to change by more than $4 \mathrm{ft}$ in any 24-hour period and by not more than $6 \mathrm{ft}$ in any 48-hour period. In dry seasons (August through March), the reservoir surface elevation is not allowed to change by more than $0.5 \mathrm{ft}$ in any 24-hour period if the elevation is lower than $6,733 \mathrm{ft}$. If the elevation is at least $6,733 \mathrm{ft}$, the elevation is not allowed to change by more than $10 \mathrm{ft}$ in any 24-hour period or by more than $15 \mathrm{ft}$ in any 48-hour period. The data presented in the last four columns of Table 6 (Crystal Reservoir) were used to check the compliance of operations with these constraints. 


\subsubsection{Seasonally Adjusted Steady Flows}

Under the seasonally adjusted steady flow scenario, the reservoirs would have steady releases, i.e., no hourly fluctuations. The daily operational model was used to simulate the seasonally adjusted steady flow scenario on the basis of the recorded inflow to the reservoir farthest upstream (Blue Mesa) and side inflows to Morrow Point and Crystal Reservoirs. Releases from each reservoir were initially assumed to be steady flows equal to the monthly releases shown in Tables 2 through 4 . Steady releases from all three reservoirs were feasible for 1987 and 1989 without violating restrictions on short-term water surface fluctuation for Crystal Reservoir. However, shifting the daily releases from Crystal Reservoir, but maintaining the average monthly releases, was necessary for April through July 1983; the adjusted daily flows are listed in Table 7.

\subsubsection{Seasonally Adjusted High Fluctuating Flows}

The seasonally adjusted high fluctuating flow scenario can be considered a variation of the seasonally adjusted steady flow scenario. Daily reservoir release volumes would be the same for both scenarios, but hourly fluctuations within the day would be maximized under the seasonally adjusted high fluctuating flow scenario.

The hourly operational model was used to test the feasibility of fluctuating hourly flows for the seasonally adjusted high fluctuating flow scenario. This model is an extension of the daily operational model and reproduces its end-of-day reservoir storages and elevations. Results from the hourly operational model indicate that releases from Blue Mesa could fluctuate up to $100 \%$ of the power plant capacity $(3,700 \mathrm{cfs})$ on any day except for those months in which minimum releases greater than zero would be required because of the large total volumes to be released. Morrow Point releases could fluctuate up to $100 \%$ of power plant capacity (5,300 cfs) during the dry season (August through March) and up to 25 to $40 \%$, depending on the year and the month, during the wet season (April through July) because of more stringent constraints on the surface fluctuation of Crystal Reservoir (Table 8). Ramp up and down are assumed to occur in one hour; ramp up is assumed to start on the hour at a certain hour such that the approximate center of the on-peak period is at 4:00 p.m. If the on-peak period is greater than 15 hours (8:00 a.m. to 11:00 p.m.), a base flow is added and the maximum fluctuation is reduced so that the peak release does not exceed the power plant capacity. The resulting release patterns for Blue Mesa and Morrow Point Reservoirs are presented in Tables 9 and 10.

\section{RESERVOIR SURFACE FLUCTUATIONS}

End-of-month reservoir elevations and daily elevation changes within each month for a moderate hydrologic year (1987) are presented in Table 2 for the seasonally adjusted steady flow scenario. The elevation changes were calculated from the reservoir storageelevation relationships shown in Table 5. Day-to-day elevation changes would range from 
The calibrated model was used to estimate water surface elevations below Morrow Point Dam for flows ranging from 100 to $5,300 \mathrm{cfs}$ (Figure 4). The water surface elevation just below the dam might fluctuate by about $10 \mathrm{ft}$ for dam releases ranging from 0 to $5,300 \mathrm{cfs}$. At $0.25 \mathrm{mi}$ below the dam, the fluctuation in elevation would be reduced to about $5.5 \mathrm{ft}$ because of the effect of Crystal Reservoir; at $0.5 \mathrm{mi}$ below the dam, the elevation would be largely controlled by Crystal Reservoir. The elevations in Figure 4 were estimated by assuming a Crystal Reservoir elevation of 6,750 ft. In the moderate water year (1987), Crystal Reservoir end-of-month elevations during the growing season (May through September) could range from about 6,747 to 6,751 $\mathrm{ft}$ (Table 2). A lower Crystal elevation would tend to lower all water surface profiles, with lesser effects on the profiles for higher flows and lesser effects on locations farther upstream.

Changes in river surface widths due to changes in flows can be estimated by using estimated water surface elevation changes from Figure 4 and land slopes from topographic maps. Because of the limitations in data used to develop the water surface profile model for the Gunnison River, estimated changes in river elevations are believed to be more reliable than the water surface elevations.

\section{CONCLUSIONS}

Three reservoir operational models were developed for the simulation of linked operations of the Aspinall Unit reservoirs. The three models were used to simulate reservoir operations on monthly, daily, and hourly bases, respectively. The models provided an efficient method for developing feasible hydropower operational scenarios for the three closely related Aspinall Unit reservoirs and also provided a tool for estimating the effects of hydropower operations on reservoir surface fluctuations.

The HEC-2 water surface profile model developed for this study provided useful information on water surface elevations downstream of Morrow Point Dam. These data were used for evaluating the potential impacts of hydropower operations on natural resources. 


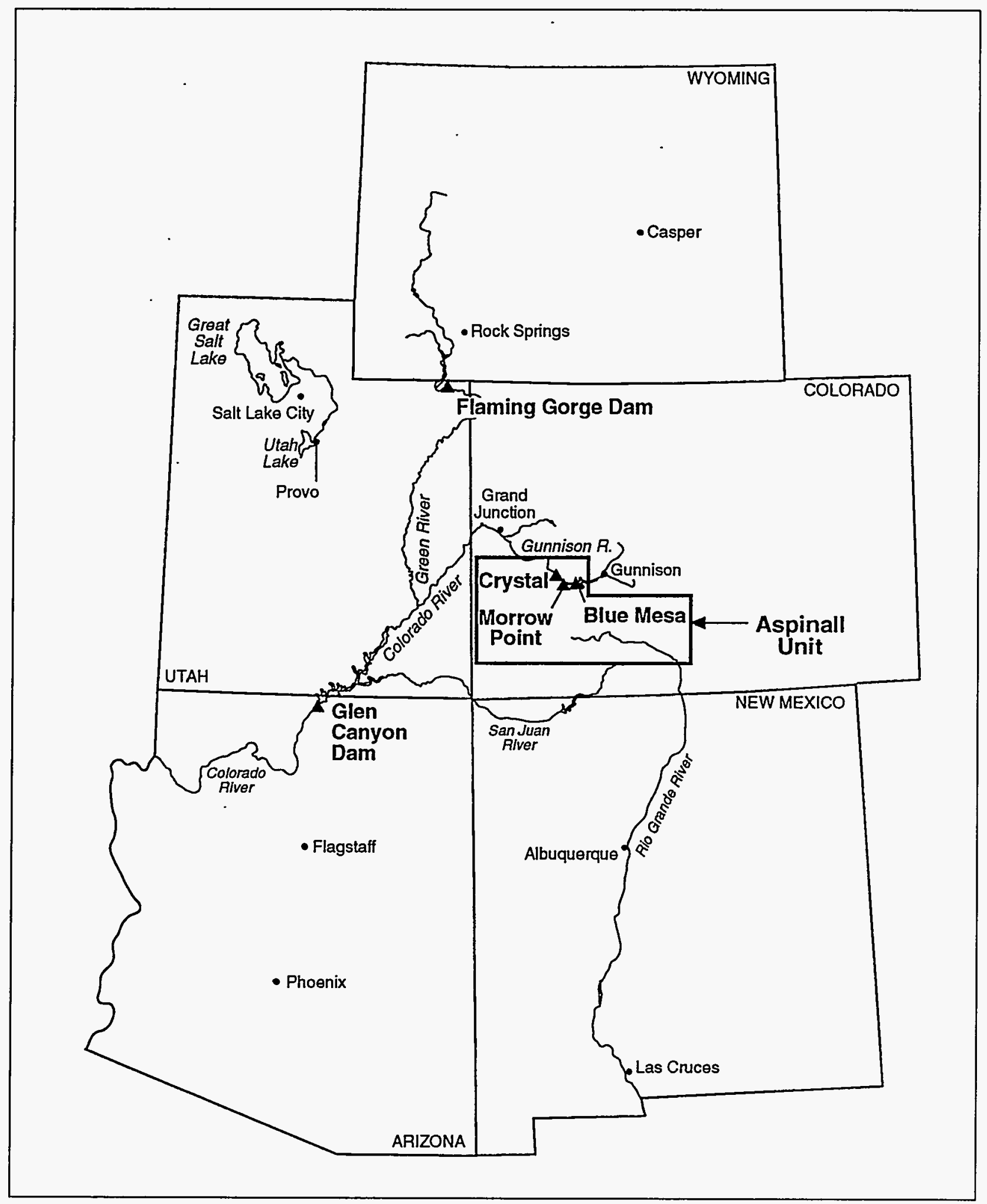

FIGURE 1 Locations of Flaming Gorge Dam, Glen Canyon Dam, and the Aspinall Unit 
8

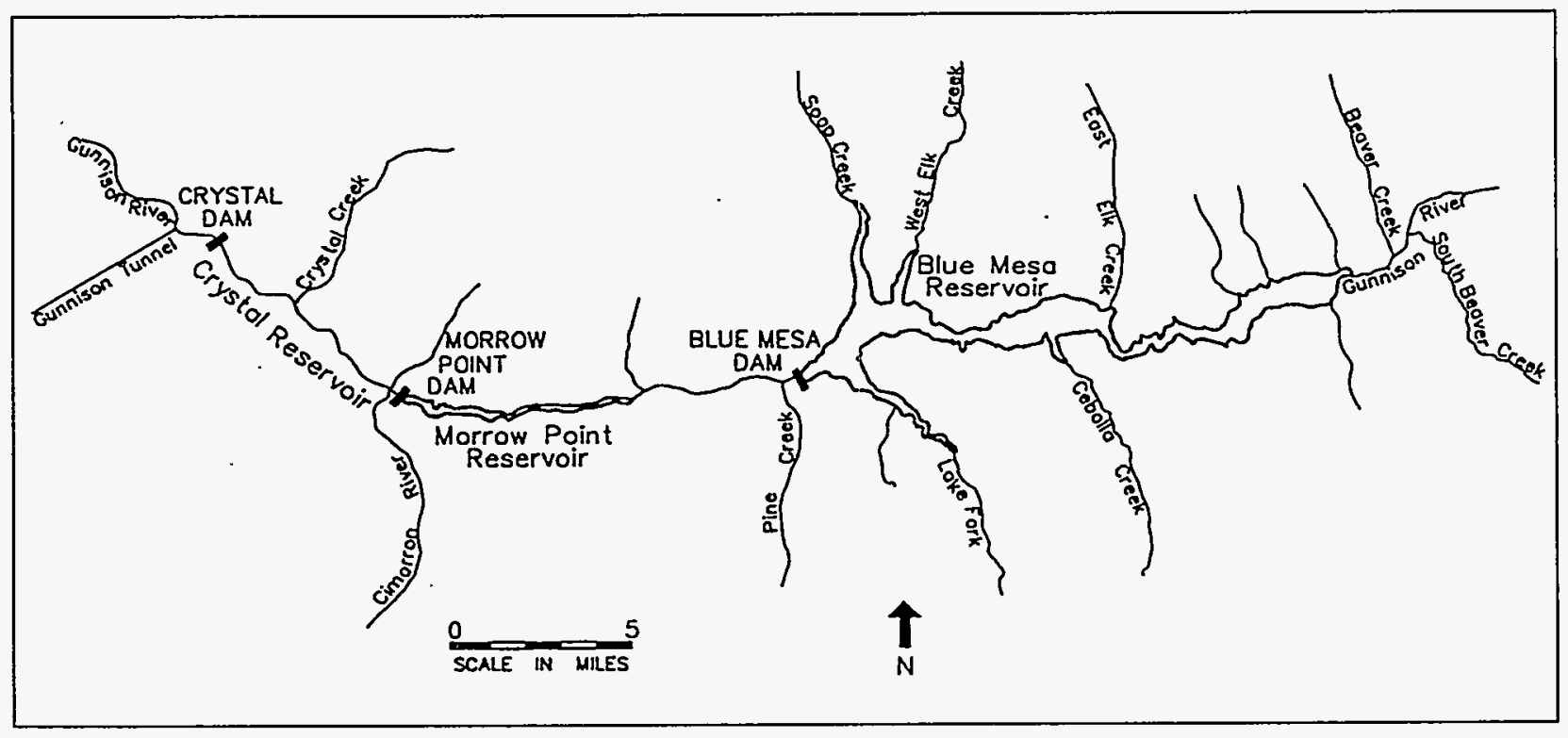

FIGURE 2 Locations of Blue Mesa, Morrow Point, and Crystal Reservoirs on the Gunnison River, Colorado 

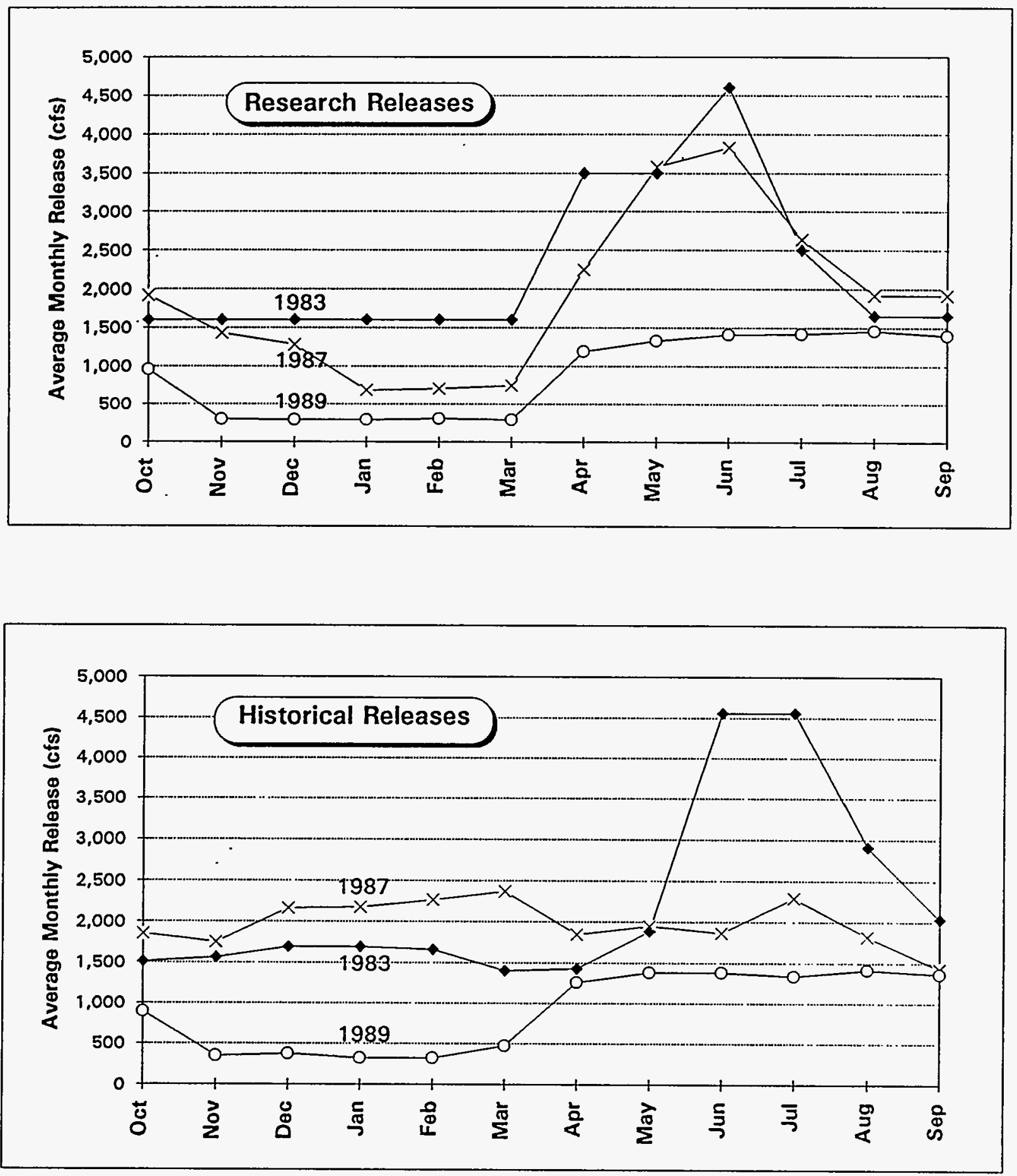

FIGÜRE 3 Research and Historical Releases at Crystal Reservoir 


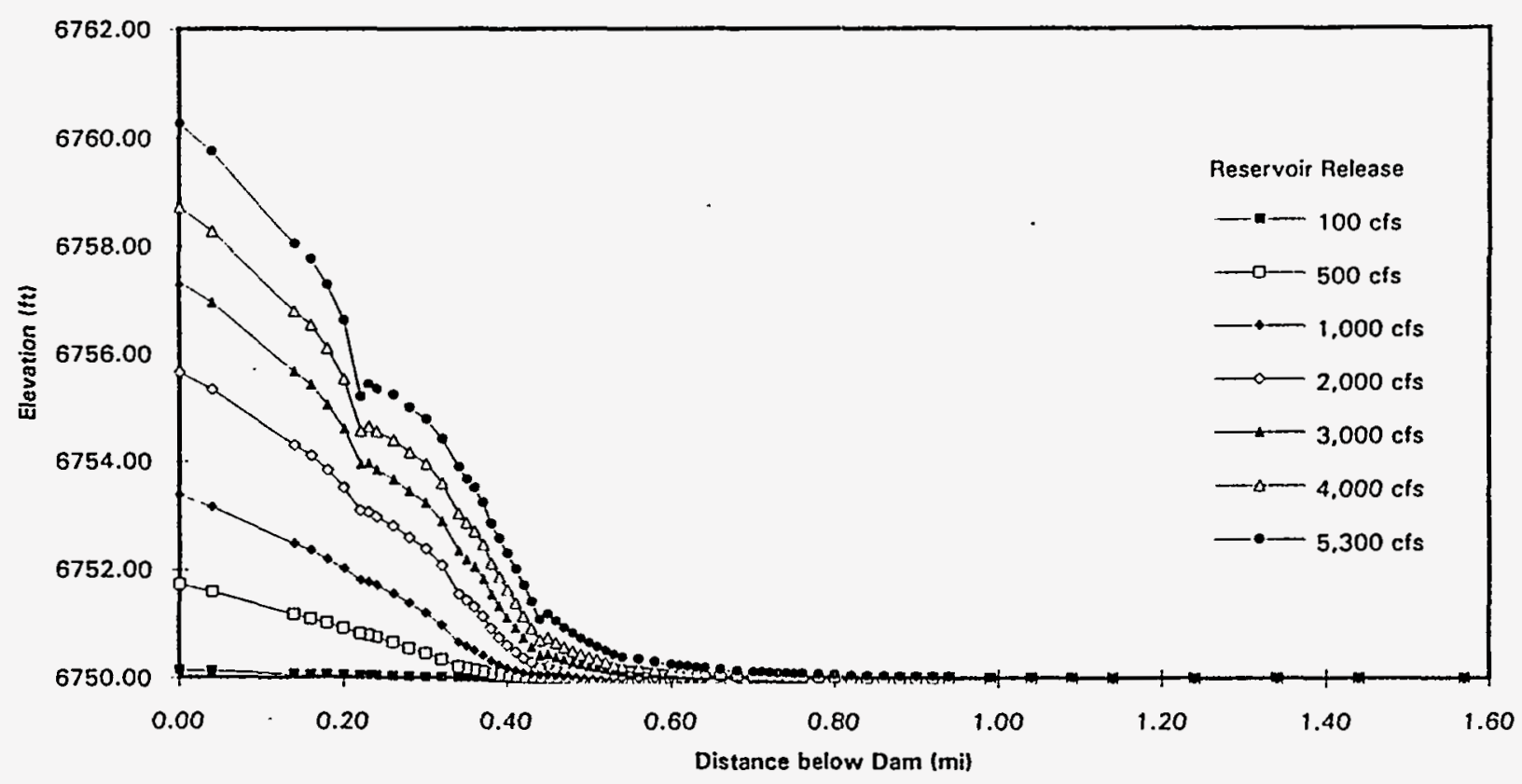

FIGURE 4 Water Surface Elevations below Morrow Point Dam (Crystal Reservoir elevation at $6,750 \mathrm{ft}$ ) 
TABLE 1 Monthly Releases from Crystal Reservoir for Moderate, Dry, and Wet Years

\begin{tabular}{cccc}
\hline & \multicolumn{3}{c}{ Daily Release (cfs) } \\
\cline { 2 - 4 } Month & Moderate Year & Dry Year & Wet Year \\
\hline Oct & 1,920 & 960 & 1,600 \\
Nov & 1,430 & 303 & 1,600 \\
Dec & 1,280 & 293 & 1,600 \\
Jan & 683 & 293 & 1,600 \\
Feb & 702 & 306 & 1,600 \\
Mar & 748 & 293 & 1,600 \\
Apr & 2,250 & 1,190 & 3,500 \\
May & 3,580 & 1,330 & 3,500 \\
Jun & 3,830 & 1,410 & 4,600 \\
Jul & 2,640 & 1,420 & 2,500 \\
Aug & 1,920 & 1,460 & 1,650 \\
Sep & 1,920 & 1,400 & 1,650 \\
---------- \\
Annual & 1,910 & 891 & 2,250 \\
\hline
\end{tabular}


TABLE 2 Reservoir Operations for the Seasonally Adjusted Steady Flow Scenario at the Aspinall Unit during a Moderate Year

\begin{tabular}{|c|c|c|c|c|c|c|c|c|c|c|c|}
\hline \multirow[b]{3}{*}{$\begin{array}{c}\text { Reservoir/ } \\
\text { Month }\end{array}$} & \multirow{2}{*}{\multicolumn{2}{|c|}{ Inflow }} & \multirow{3}{*}{$\begin{array}{c}\text { Side } \\
\text { Inflow } \\
\text { (cfs) }\end{array}$} & \multicolumn{3}{|c|}{ Release } & \multirow[b]{3}{*}{$\begin{array}{c}\text { Evaporation } \\
\text { (cfs) }\end{array}$} & \multirow{3}{*}{$\begin{array}{c}\text { Ending } \\
\text { Storage } \\
\text { (acre-feet) }\end{array}$} & \multirow{3}{*}{$\begin{array}{l}\text { Ending } \\
\text { Elevation } \\
\text { (ft) }\end{array}$} & \multirow{2}{*}{\multicolumn{2}{|c|}{ Elevation Change }} \\
\hline & & & & & & & & & & & \\
\hline & acre-feet & $\mathrm{cfs}$ & & $\begin{array}{c}\text { Power } \\
\text { (cfs) }\end{array}$ & $\begin{array}{l}\text { Other } \\
(\mathrm{cfs})\end{array}$ & $\begin{array}{l}\text { Total } \\
\text { (cfs) }\end{array}$ & & & & $\mathrm{ft}$ & $\mathrm{ft} / \mathrm{d}$ \\
\hline \multicolumn{12}{|l|}{ Blue Mesa ${ }^{a}$} \\
\hline Oct & 62,539 & 1,017 & 0 & 1,570 & 0 & 1,570 & 8.8 & 625,462 & $7,495.61$ & -4.25 & -0.14 \\
\hline Nov & 51,278 & 862 & 0 & 1,200 & 0 & 1,200 & 4.2 & 605,086 & $7,493.05$ & -2.56 & -0.09 \\
\hline Dec & 30,550 & 497 & 0 & 1,050 & 0 & 1,050 & 2.5 & 570,920 & $7,488.69$ & -4.37 & -0.14 \\
\hline Jan & 27,811 & 452 & 0 & 500 & 0 & 500 & 2.0 & 567,864 & $7,488.29$ & -0.40 & -0.01 \\
\hline Feb & 28,728 & 517 & 0 & 510 & 0 & 510 & 2.6 & 568,124 & $7,488.33$ & 0.03 & 0.00 \\
\hline Mar & 55,524 & 903 & 0 & 500 & 0 & 500 & 4.9 & 592,603 & $7,491.47$ & 3.14 & 0.10 \\
\hline Apr & 125,807 & 2,114 & 0 & 1,600 & 0 & 1,600 & 7.8 & 622,739 & $7,495.27$ & 3.80 & 0.13 \\
\hline May & 271,476 & 4,415 & 0 & 2,370 & 0 & 2,370 & 14.6 & 747,592 & $7,510.23$ & 14.96 & 0.48 \\
\hline Jun & 255,805 & 4,299 & 0 & 3,050 & 0 & 3,050 & 22.8 & 820,553 & $7,518.42$ & 8.19 & 0.27 \\
\hline Jul & 97,192 & 1,581 & 0 & 2,350 & 0 & 2,350 & 24.6 & 771,736 & $7,512.98$ & -5.43 & -0.18 \\
\hline Aug & 64,634 & 1,051 & 0 & 1,750 & 0 & 1,750 & 18.6 & 727,623 & $7,507.91$ & -5.07 & -0.16 \\
\hline Sep & 44,320 & 745 & 0 & 1,750 & 0 & 1,750 & 15.2 & 666,907 & $7,500.70$ & -7.21 & -0.24 \\
\hline \multicolumn{12}{|c|}{ Morrow Point ${ }^{b}$} \\
\hline Oct & 96,535 & 1,570 & 94 & 1,700 & 0 & 1,700 & 0.0 & 112,632 & $7,154.30$ & -2.75 & -0.09 \\
\hline Nov & 71,405 & 1,200 & 61 & 1,280 & 0 & 1,280 & 0.0 & 111,489 & $7,152.86$ & -1.44 & -0.05 \\
\hline Dec & 64,562 & 1,050 & 69 & 1,100 & 0 & 1,100 & 0.0 & 112,627 & $7,154.29$ & 1.44 & 0.05 \\
\hline Jan & 30,744 & 500 & 61 & 570 & 0 & 570 & 0.0 & 112,061 & $7,153.58$ & -0.71 & -0.02 \\
\hline Feb & 28,324 & 510 & 82 & 580 & 0 & 580 & 0.0 & 112,744 & $7,154.44$ & 0.86 & 0.03 \\
\hline Mar & 30,744 & 500 & 98 & 650 & 0 & 650 & 0.0 & 109,528 & $7,150.36$ & -4.08 & -0.13 \\
\hline $\mathrm{Apr}$ & 95,207 & 1,600 & 373 & 1,970 & 0 & 1,970 & 0.0 & 109,707 & $7,150.59$ & 0.23 & 0.01 \\
\hline May & 145,726 & 2,370 & 534 & 2,890 & 0 & 2,890 & 2.1 & 110,439 & $7,151.52$ & 0.94 & 0.03 \\
\hline Jun & 181,488 & 3,050 & 311 & 3,325 & 0 & 3,325 & 14.3 & 111,730 & $7,153.16$ & 1.64 & 0.05 \\
\hline Jul & 144,496 & 2,350 & 74 & 2,480 & 0 & 2,480 & 13.6 & 107,420 & $7,147.63$ & -5.53 & -0.18 \\
\hline Aug & 107,603 & 1,750 & 58 & 1,770 & 0 & 1,770 & 1.4 & 109,688 & $7,150.56$ & 2.93 & 0.09 \\
\hline Sep & 104,132 & 1,750 & 44 & 1,820 & 0 & 1,820 & 0.0 & 108,124 & $7,148.55$ & -2.02 & -0.07 \\
\hline
\end{tabular}


TABLE 2 (Cont.)

\begin{tabular}{|c|c|c|c|c|c|c|c|c|c|c|c|}
\hline \multirow{3}{*}{$\begin{array}{l}\text { Reservoir/ } \\
\text { Month }\end{array}$} & \multirow{2}{*}{\multicolumn{2}{|c|}{ Inflow }} & \multirow{3}{*}{$\begin{array}{l}\text { Side } \\
\text { Inflow } \\
\text { (cfs) }\end{array}$} & \multicolumn{3}{|c|}{ Release } & \multirow{3}{*}{$\begin{array}{c}\text { Evaporation } \\
\text { (cfs) }\end{array}$} & \multirow{3}{*}{$\begin{array}{c}\text { Ending } \\
\text { Storage } \\
\text { (acre-feet) }\end{array}$} & \multirow{3}{*}{$\begin{array}{c}\text { Ending } \\
\text { Elevation } \\
\cdot(\mathrm{ft})\end{array}$} & \multirow{2}{*}{\multicolumn{2}{|c|}{ Elevation Change }} \\
\hline & & & & & & & & & & & \\
\hline & acre-feet & cfs & & $\begin{array}{l}\text { Fower } \\
\text { (cfs) }\end{array}$ & $\begin{array}{l}\text { (cfs) } \\
\text { (cfiser }\end{array}$ & $\begin{array}{l}\text { Total } \\
\text { (cfs) }\end{array}$ & & & & $\mathrm{ft}$ & $\mathrm{ft} / \mathrm{d}$ \\
\hline \multicolumn{12}{|l|}{ Crystale } \\
\hline Oct & 104,529 & 1,700 & 210 & 1,763 & 156 & 1,919 & 0.0 & 15,276 & $6,747.04$ & -1.96 & -0.06 \\
\hline Nov & 76,165 & 1,280 & 135 & 1,429 & 0 & 1,429 & 0.0 & 14,443 & $6,744.02$ & -3.02 & -0.10 \\
\hline Dec & 67,636 & 1,100 & 152 & 1,285 & 0 & 1,285 & 0.0 & 12,413 & $6,736.29$ & -7.73 & -0.25 \\
\hline Jan & 35,048 & 570 & 135 & 683 & 0 & 683 & 0.0 & 13,766 & $6,741.50$ & 5.21 & 0.17 \\
\hline $\mathrm{Feb}$ & 32,212 & 580 & 123 & 702 & 0 & 702 & 0.0 & 13,822 & $6,741.71$ & 0.21 & 0.01 \\
\hline Mar & 39,967 & 650 & 147 & 748 & 0 & 748 & 0.0 & 16,835 & $6,752.48$ & 10.77 & 0.35 \\
\hline Apr & 117,223 & 1,970 & 275 & 1,763 & 489 & 2,252 & 0.0 & 16,418 & $6,751.05$ & -1.43 & .0 .05 \\
\hline May & 177,699 & 2,890 & 680 & 1,763 & 1,815 & 3,578 & 0.0 & 15,926 & $6,749.34$ & -1.71 & -0.06 \\
\hline Jun & 197,851 & 3,325 & 507 & 1,763 & 2,069 & 3,832 & 0.0 & 15,926 & $6,749.34$ & 0.00 & 0.00 \\
\hline Jul & 152,489 & 2,480 & 164 & 1,763 & 872 & 2,635 & 0.0 & 16,480 & $6,751.26$ & 1.92 & 0.06 \\
\hline Aug & 108,833 & 1,770 & 130 & 1,763 & 156 & 1,919 & 0.0 & 15,311 & $6,747.16$ & -4.10 & -0.13 \\
\hline Sep & 108,297 & 1,820 & 97 & 1,763 & 153 & 1,916 & 0.0 & 15,395 & $6,747.46$ & 0.30 & 0.01 \\
\hline
\end{tabular}

a Starting conditions: storage, 660,000 acre-feet; elevation, 7,499.86 ft. Totals (acre-feet): inflow, 1,115,664; release, 1,100,965; evaporation, 7,793 .

b Starting conditions: storage, 114,833 acre-feet; elevation, 7,157.05 ft. Totals (acre-feet): inflow, 1,100,965; side inflow, 112,178; release, 1,217,950; evaporation, 1,902.

c Starting conditions: storage, 15,829 acre-feet; elevation, 6,749.00 ft. Totals (acre-feet): inflow, 1,217,950; side inflow, 166,679; release, $1,385,063$; evaporation, 0 . 
TABLE 3 Reservoir Operations for the Seasonally Adjusted Steady Flow Scenario at the Aspinall Unit during a Dry Year

\begin{tabular}{|c|c|c|c|c|c|c|c|c|c|c|c|}
\hline \multirow{3}{*}{$\begin{array}{c}\text { Reservoir/ } \\
\text { Month }\end{array}$} & \multirow{2}{*}{\multicolumn{2}{|c|}{ Inflow }} & \multirow{3}{*}{$\begin{array}{l}\text { Side } \\
\text { Inflow } \\
\text { (cfs) }\end{array}$} & \multicolumn{3}{|c|}{ Release } & \multirow[b]{3}{*}{$\begin{array}{c}\text { Evaporation } \\
\text { (cfs) }\end{array}$} & \multirow{3}{*}{$\begin{array}{c}\text { Ending } \\
\text { Storage } \\
\text { (acre-feet) }\end{array}$} & \multirow{3}{*}{$\begin{array}{l}\text { Ending } \\
\text { Elevation } \\
\text { (ft) }\end{array}$} & \multirow{2}{*}{\multicolumn{2}{|c|}{ Elevation Change }} \\
\hline & & & & & & & & & & & \\
\hline & acre-feet & $\mathrm{cfs}$ & & $\begin{array}{c}\text { Power } \\
\text { (cfs) }\end{array}$ & $\begin{array}{l}\text { Other } \\
\text { (cfs) }\end{array}$ & $\begin{array}{l}\text { Total } \\
\text { (cfs) }\end{array}$ & & & & $\mathrm{ft}$ & $\mathrm{ft} / \mathrm{d}$ \\
\hline \multicolumn{12}{|l|}{ Blue Mesa ${ }^{\alpha}$} \\
\hline Oct & 30,258 & 492 & 0 & 650 & 0 & 650 & 6.4 & 439,014 & $7,470.52$ & -1.48 & -0.05 \\
\hline Nov & 26,684 & 448 & 0 & 180 & 0 & 180 & 3.2 & 454,796 & $7,472.82$ & 2.30 & 0.08 \\
\hline Dec & 23,654 & 385 & 0 & 150 & 0 & 150 & 2.0 & 469,104 & $7,474.88$ & 2.05 & 0.07 \\
\hline Jan & 27,006 & 439 & 0 & 200 & 0 & 200 & 2.0 & 483,690 & $7,476.94$ & 2.06 & 0.07 \\
\hline Feb & 23,941 & 431 & 0 & 250 & 0 & 250 & 2.8 & 493,591 & $7,478.32$ & 1.38 & 0.05 \\
\hline Mar & 44,848 & 729 & 0 & 200 & 0 & 200 & 6.2 & 525,760 & $7,482.73$ & 4.40 & 0.14 \\
\hline Apr & 96,499 & 1,622 & 0 & 760 & 0 & 760 & 10.8 & 576,394 & $7,489.39$ & 6.67 & 0.22 \\
\hline May & 124,997 & 2,033 & 0 & 900 & 0 & 900 & 16.0 & $.645,068$ & $7,498.03$ & 8.64 & 0.28 \\
\hline Jun & 123,561 & 2,077 & 0 & 1,150 & 0 & 1,150 & 21.4 & 698,926 & $7,504.53$ & 6.50 & 0.22 \\
\hline Jul & 59,496 & 968 & 0 & 1,300 & 0 & 1,300 & 22.2 & 677,123 & $7,501.93$ & -2.60 & -0.08 \\
\hline Aug & 56,234 & 915 & 0 & 1,300 & 0 & 1,300 & 17.2 & 652,366 & $7,498.93$ & -3.00 & -0.10 \\
\hline Sep & 27,920 & 469 & 0 & 1,300 & 0 & 1,300 & 13.6 & 602,121 & $7,492.68$ & -6.25 & -0.21 \\
\hline \multicolumn{12}{|c|}{ Morrow Point ${ }^{b}$} \\
\hline Oct & 39,967 & 650 & 38 & 720 & 0 & 720 & 0.0 & 112,021 & $7,153.53$ & -2.47 & -0.08 \\
\hline Nov & 10,711 & 180 & 37 & 200 & 0 & 200 & 0.0 & 113,033 & $7,154.80$ & 1.27 & 0.04 \\
\hline Dec & 9,223 & 150 & 37 & 200 & 0 & 200 & 0.0 & 112,234 & $7,153.80$ & -1.01 & -0.03 \\
\hline Jan & 12,298 & 200 & (15) & 220 & 0 & 220 & 0.0 & 110,082 & $7,151.07$ & -2.73 & -0.09 \\
\hline Feb & 13,884 & 250 & 24 & 270 & 0 & 270 & 0.0 & 110,304 & $7,151.35$ & 0.28 & 0.01 \\
\hline Mar & 12,298 & 200 & 66 & 260 & 0 & 260 & 0.0 & 110,673 & $7,151.82$ & 0.47 & 0.02 \\
\hline Apr & 45,223 & 760 & 207 & 970 & 0 & 970 & 0.0 & 110,494 & $7,151.59$ & -0.23 & -0.01 \\
\hline May & 55,339 & 900 & 192 & 1,090 & 0 & 1,090 & 2.1 & 110,488 & $7,151.58$ & -0.01 & 0.00 \\
\hline Jun & 68,430 & 1,150 & 102 & 1,225 & 0 & 1,225 & 14.3 & 111,244 & $7,152.55$ & 0.96 & 0.03 \\
\hline Jul & 79,934 & 1,300 & 37 & 1,350 & 0 & 1,350 & 13.8 & 109,596 & $7,150.44$ & -2.10 & -0.07 \\
\hline Aug & 79,934 & 1,300 & 88 & 1,400 & 0 & 1,400 & 1.4 & 108,772 & $7,149.38$ & -1.06 & -0.03 \\
\hline Sep & 77,355 & 1,300 & 63 & 1,300 & 0 & 1,300 & 0.0 & 112,521 & $7,154.16$ & 4.78 & 0.16 \\
\hline
\end{tabular}


TABLE 3 (Cont.)

\begin{tabular}{|c|c|c|c|c|c|c|c|c|c|c|c|}
\hline \multirow[b]{3}{*}{$\begin{array}{l}\text { Reservoir/ } \\
\text { Month }\end{array}$} & \multirow{2}{*}{\multicolumn{2}{|c|}{ Inflow }} & \multirow{3}{*}{$\begin{array}{c}\text { Side } \\
\text { Inflow } \\
\text { (cfs) }\end{array}$} & \multicolumn{3}{|c|}{ Release } & \multirow[b]{3}{*}{$\begin{array}{c}\text { Evaporation } \\
\text { (cfs) }\end{array}$} & \multirow{3}{*}{$\begin{array}{c}\text { Ending } \\
\text { Storage } \\
\text { (acre-feet) }\end{array}$} & \multirow{3}{*}{$\begin{array}{l}\text { Ending } \\
\text { Elevation } \\
\text { (ft) }\end{array}$} & \multirow{2}{*}{\multicolumn{2}{|c|}{ Elevation Change }} \\
\hline & & & & & & & & & & & \\
\hline & acre-feet & $\mathrm{cfs}$ & & $\begin{array}{l}\text { Power } \\
\text { (cfs) }\end{array}$ & $\begin{array}{c}\text { Other } \\
\text { (cfs) }\end{array}$ & $\begin{array}{l}\text { Total } \\
\text { (cfs) }\end{array}$ & & & & $\mathrm{ft}$ & $\mathrm{ft} / \mathrm{d}$ \\
\hline \multicolumn{12}{|l|}{ Crystal } \\
\hline Oct & 44,271 & 720 & 186 & 960 & 0 & 960 & 0.0 & 13,657 & $6,741.09$ & -11.87 & -0.38 \\
\hline Nov & 11,901 & 200 & 98 & 303 & 0 & 303 & 0.0 & 13,359 & $6,739.96$ & -1.13 & -0.04 \\
\hline Dec & 12,298 & 200 & 92 & 293 & 0 & 293 & 0.0 & 13,298 & $6,739.72$ & -0.23 & -0.01 \\
\hline Jan & 13,527 & 220 & 73 & 293 & 0 & 293 & 0.0 & 13,298 & $6,739.72$ & 0.00 & 0.00 \\
\hline Feb & 14,995 & 270 & 46 & 306 & 0 & 306 & 0.0 & 13,853 & $6,741.83$ & 2.10 & 0.08 \\
\hline Mar & 15,987 & 260 & 99 & 293 & 0 & 293 & 0.0 & 17,911 & $6,756.08$ & 14.25 & 0.46 \\
\hline Apr & 57,719 & 970 & 224 & 1,193 & 0 & 1,193 & 0.0 & 17,971 & $6,756.27$ & 0.20 & 0.01 \\
\hline May & 67,021 & 1,090 & 244 & 1,334 & 0 & 1,334 & 0.0 & 17,971 & $6,756.27$ & 0.00 & 0.00 \\
\hline Jun & 72,893 & 1,225 & 183 & 1,412 & 0 & 1,412 & 0.0 & 17,733 & $6,755.49$ & -0.78 & -0.03 \\
\hline Jul & 83,008 & 1,350 & 64 & 1,415 & 0 & 1,415 & 0.0 & 17,671 & $6,755.29$ & -0.20 & -0.01 \\
\hline Aug & 86,083 & 1,400 & 43 & 1,464 & 0 & 1,464 & 0.0 & 16,380 & $6,750.92$ & -4.37 & -0.14 \\
\hline Sep & 77,355 & 1,300 & 66 & 1,395 & 0 & 1,395 & 0.0 & 14,654 & $6,744.79$ & -6.13 & -0.20 \\
\hline
\end{tabular}

a Starting conditions: storage, 449,116 acre-feet; elevation, 7,472.00 ft. Totals (acre-feet): inflow, 665,098; release, 504,595; evaporation, 7,498.

b Starting conditions: storage, 113,989 acre-feet; elevation, 7,156.00 ft. Totals (acre-feet): inflow, 504,595; side inflow, 52,909; release, 557,058; evaporation, 1,915.

c Starting conditions: storage, 16,977 acre-feet; elevation, 6,752.96 ft. Totals (acre-feet): inflow, 557,058; side inflow, 85,783; release, 645,163; evaporation, 0 . 
TABLE 4 Reservoir Operations for the Seasonally Adjusted Steady Flow Scenario at the Aspinall Unit during a Wet Year

\begin{tabular}{|c|c|c|c|c|c|c|c|c|c|c|c|}
\hline \multirow[b]{3}{*}{$\begin{array}{l}\text { Reservoir/ } \\
\text { Month }\end{array}$} & \multirow{2}{*}{\multicolumn{2}{|c|}{ Inflow }} & \multirow{3}{*}{$\begin{array}{c}\text { Side } \\
\text { Inflow } \\
\text { (cfs) }\end{array}$} & \multicolumn{3}{|c|}{ Release } & \multirow[b]{3}{*}{$\begin{array}{l}\text { Evaporation } \\
\text { (cfs) }\end{array}$} & \multirow{3}{*}{$\begin{array}{c}\text { Ending } \\
\text { Storage } \\
\text { (acre-feet) }\end{array}$} & \multirow{3}{*}{$\begin{array}{l}\text { Ending } \\
\text { Elevation } \\
\text { (ft) }\end{array}$} & \multirow{2}{*}{\multicolumn{2}{|c|}{ Elevation Change }} \\
\hline & & & & \multirow[b]{2}{*}{$\begin{array}{c}\text { Power } \\
\text { (cfs) }\end{array}$} & \multirow[b]{2}{*}{$\begin{array}{l}\text { Other } \\
\text { (cfs) }\end{array}$} & \multirow[b]{2}{*}{$\begin{array}{l}\text { Total } \\
\text { (cfs) }\end{array}$} & & & & & \\
\hline & acre-feet & $\mathrm{cfs}$ & & & & & & & & $\mathrm{ft}$ & $\mathrm{ft} / \mathrm{d}$ \\
\hline Blue Mesa ${ }^{\alpha}$ & & & & . & & & & & & & \\
\hline Oct & 64,331 & 1,046 & 0 & 1,320 & 0 & 1,320 & 8.9 & 721,799 & $7,507.23$ & -2.02 & -0.07 \\
\hline Nov & 36,661 & 616 & 0 & 1,400 & 0 & 1,400 & 4.2 & 674,905 & $7,501.66$ & -5.56 & -0.19 \\
\hline Dec & 29,191 & 475 & 0 & 1,500 & 0 & 1,500 & 2.7 & 611,698 & $7,493.89$ & -7.78 & -0.25 \\
\hline Jan & 29,301 & 477 & 0 & 1,540 & 0 & 1,540 & 2.0 & 546,185 & $7,485.45$ & -8.43 & -0.27 \\
\hline Feb & 25,993 & 468 & 0 & 1,470 & 0 & 1,470 & 3.3 & 490,355 & $7,477.87$ & -7.58 & -0.27 \\
\hline Mar & 42,347 & 689 & 0 & 1,450 & 0 & 1,450 & 5.9 & 443,183 & $7,471.13$ & -6.74 & -0.22 \\
\hline Apr & 58,197 & 978 & 0 & 3,220 & 0 & 3,220 & 9.5 & 309,211 & $7,449.49$ & -21.65 & -0.72 \\
\hline May & 164,542 & 2,676 & 0 & 2,200 & 0 & 2,200 & 12.0 & 337,743 & $7,454.48$ & 4.99 & 0.16 \\
\hline Jun & 398,810 & 6,702 & 0 & 2,330 & 0 & 2,330 & 22.8 & 596,551 & $7,491.97$ & 37.49 & 1.25 \\
\hline Jul & 218,498 & 3,554 & 0 & 1,550 & 0 & 1,550 & 25.2 & 718,194 & $7,506.81$ & 14.84 & 0.48 \\
\hline Aug & 123,580 & 2,010 & 0 & 1,100 & 0 & 1,100 & 19.7 & 772,927 & $7,513.12$ & 6.31 & 0.20 \\
\hline Sep & 58,040 & 975 & 0 & 1,500 & 0 & 1,500 & 15.8 & 740,770 & $7,509.43$ & -3.68 & -0.12 \\
\hline Morrow Poir & & & & & & & & & & & \\
\hline Oct & 81,164 & 1,320 & 81 & 1,410 & 0 & 1,410 & 0.0 & 112,214 & $7,153.77$ & -0.70 & -0.02 \\
\hline Nov & 83,306 & 1,400 & 37 & 1,510 & 0 & 1,510 & 0.0 & 107,870 & $7,148.22$ & -5.55 & -0.19 \\
\hline Dec & 92,231 & 1,500 & 37 & 1,520 & 0 & 1,520 & 0.0 & 108,915 & $7,149.57$ & 1.35 & 0.04 \\
\hline Jan & 94,691 & 1,540 & 35 & 1,520 & 0 & 1,520 & 0.0 & 112,297 & $7,153.88$ & 4.31 & 0.14 \\
\hline Feb & 81,640 & 1,470 & 46 & 1,540 & 0 & 1,540 & 0.0 & 110,964 & $7,152.19$ & -1.69 & -0.06 \\
\hline Mar & 89,157 & 1,450 & 68 & 1,535 & 0 & 1,535 & 0.0 & 109,919 & $7,150.86$ & -1.33 & -0.04 \\
\hline Apr & 191,603 & 3,220 & 141 & 3,345 & 0 & 3,345 & 0.0 & 110,871 & $7,152.07$ & 1.21 & 0.04 \\
\hline May & 135,273 & 2,200 & 581 & 2,765 & 0 & 2,765 & 2.1 & 111,725 & $7,153.16$ & 1.08 & 0.03 \\
\hline Jun & 138,645 & 2,330 & 855 & 3,180 & 0 & 3,180 & 14.3 & 111,172 & $7,152.45$ & -0.70 & -0.02 \\
\hline Jul & 95,306 & 1,550 & 298 & 1,840 & 0 & 1,840 & 13.6 & 110,828 & $7,152.02$ & -0.44 & -0.01 \\
\hline Aug & 67,636 & 1,100 & 152 & 1,300 & 0 & 1,300 & 1.4 & 107,790 & $7,148.12$ & -3.90 & -0.13 \\
\hline Sep & 89,256 & 1,500 & 60 & 1,510 & 0 & 1,510 & 0.0 & 110,765 & $7,151.94$ & 3.82 & 0.13 \\
\hline
\end{tabular}


TABLE 4 (Cont.)

\begin{tabular}{|c|c|c|c|c|c|c|c|c|c|c|c|}
\hline \multirow{3}{*}{$\begin{array}{c}\text { Reservoir/ } \\
\text { Month }\end{array}$} & \multirow{2}{*}{\multicolumn{2}{|c|}{ Inflow }} & \multirow{3}{*}{$\begin{array}{c}\text { Side } \\
\text { Inflow } \\
\text { (cfs) }\end{array}$} & \multicolumn{3}{|c|}{ Release } & \multirow{3}{*}{$\begin{array}{c}\text { Evaporation } \\
\text { (cfs) }\end{array}$} & \multirow{3}{*}{$\begin{array}{c}\text { Ending } \\
\text { Storage } \\
\text { (acre-feet) }\end{array}$} & \multirow{3}{*}{$\begin{array}{l}\text { Ending } \\
\text { Elevation } \\
\cdot \quad(\mathrm{ft})\end{array}$} & \multirow{2}{*}{\multicolumn{2}{|c|}{ Elevation Change }} \\
\hline & & & & & & & & & & & \\
\hline & acre-feet & cfs & & $\begin{array}{c}\text { Power } \\
\text { (cfs) }\end{array}$ & $\begin{array}{l}\text { Uther } \\
\text { (cfs) }\end{array}$ & $\begin{array}{l}\text { Total } \\
\text { (cfs) }\end{array}$ & & & & $\mathrm{ft}$ & $\mathrm{ft} / \mathrm{d}$ \\
\hline \multicolumn{12}{|l|}{ Crystal $^{c}$} \\
\hline Oct & 86,697 & 1,410 & 180 & 1,600 & 0 & 1,600 & 0.0 & 1,488 & $6,747.79$ & -2.17 & -0.07 \\
\hline Nov & 89,851 & 1,510 & 83 & 1,600 & 0 & 1,600 & 0.0 & 15,072 & $6,746.30$ & -1.49 & -0.05 \\
\hline Dec & 93,461 & 1,520 & 83 & 1,600 & 0 & 1,600 & 0.0 & 15,256 & $6,746.97$ & 0.66 & 0.02 \\
\hline Jan & 93,461 & 1,520 & 78 & 1,600 & 0 & 1,600 & 0.0 & 15,133 & $6,746.53$ & -0.44 & -0.01 \\
\hline Feb & 85,527 & 1,540 & 70 & 1,600 & 0 & 1,600 & 0.0 & 15,688 & $6,748.50$ & 1.98 & 0.07 \\
\hline Mar & 94,383 & 1,535 & 102 & 1,600 & 0 & 1,600 & 0.0 & 17,963 & $6,756.25$ & 7.75 & 0.25 \\
\hline Apr & 199,041 & 3,345 & 153 & 1,763 & 1,737 & 3,500 & 0.0 & 17,844 & $6,755.86$ & -0.39 & -0.01 \\
\hline May & 170,013 & 2,765 & 739 & 1,763 & 1,737 & 3,500 & 0.0 & 18,090 & $6,756.67$ & 0.81 & 0.03 \\
\hline Jun & 189,223 & 3,180 & 1,395 & 1,763 & 2,837 & 4,600 & 0.0 & 16,603 & $6,751.69$ & -4.98 & -0.17 \\
\hline Jul & 113,137 & 1,840 & 663 & $1,763$. & 737 & 2,500 & 0.0 & 16,787 & $6,752.32$ & 0.63 & 0.02 \\
\hline Aug & 79,934 & 1,300 & 339 & 1,650 & 0 & 1,650 & 0.0 & 16,111 & $6,749.99$ & -2.33 & -0.08 \\
\hline Sep & 89,851 & 1,510 & 133 & 1,650 & 0 & 1,650 & 0.0 & 15,694 & $6,748.53$ & -1.46 & -0.05 \\
\hline
\end{tabular}

a Starting conditions: storage, 739,179 acre-feet; elevation, 7,509.25 ft. Totals (acre-feet): inflow, 1,249,491; release, 1,239,907; evaporation, 7,993.

b Starting conditions: storage, 112,767 acre-feet; elevation, 7,154.47 ft. Totals (acre-feet): inflow, 1,239,907; side inflow, 144,575; release, 1,384,581; evaporation, 1,902.

c Starting conditions: storage, 16,103 acre-feet; elevation, 6,749.96 ft. Totals (acre-feet): inflow, 1,384,581; side inflow, 243,142; release, 1,628,131; evaporation, 0 . 
TABLE 5 Bureau of Reclamation Coefficient Tables Used to Calculate Reservoir Storages ${ }^{a}$

\begin{tabular}{|c|c|c|c|c|}
\hline Reservoir & $\begin{array}{l}\text { Elevation, } \mathrm{E}_{\mathrm{b}} \\
(\mathrm{ft})\end{array}$ & $\cdot$ & b & c \\
\hline Blue Mesa & $\begin{array}{l}7350 . \\
7380 . \\
7400 . \\
7420 . \\
7450 . \\
7470 . \\
7490 . \\
7510 . \\
7519 .\end{array}$ & $\begin{array}{r}-14174.00 \\
47203.00 \\
101405.00 \\
170210.00 \\
312210.00 \\
435478.00 \\
501135.00 \\
745605.00 \\
829523.00\end{array}$ & $\begin{array}{c}1668.82 \\
2415.18 \\
3000.20 \\
2899.79 \\
5572.05 \\
6767.88 \\
7776.96 \\
8680.00 \\
\text { b }\end{array}$ & $\begin{array}{c}12.59 \\
14.80 \\
22.13 \\
27.75 \\
29.43 \\
25.68 \\
22.50 \\
26.80 \\
\text { b }\end{array}$ \\
\hline Morrow Point & $\begin{array}{l}6770 . \\
6780 . \\
6790 . \\
6800 . \\
6810 . \\
6820 . \\
6830 . \\
6850 . \\
6860 . \\
6880 . \\
6900 . \\
6920 . \\
6960 . \\
7038 .\end{array}$ & $\begin{array}{r}0.00 \\
5.00 \\
30.00 \\
90.00 \\
195.00 \\
365.00 \\
625.00 \\
1490.00 \\
2131.07 \\
3781.61 \\
5931.25 \\
8911.77 \\
17120.46 \\
39833.16\end{array}$ & $\begin{array}{r}0.00 \\
1.00 \\
4.00 \\
8.00 \\
13.00 \\
21.00 \\
30.70 \\
56.00 \\
71.39 \\
93.09 \\
125.13 \\
175.79 \\
235.21 \\
402.63\end{array}$ & $\begin{array}{l}0.05 \\
0.15 \\
0.20 \\
0.25 \\
0.40 \\
0.50 \\
0.63 \\
0.80 \\
0.55 \\
0.73 \\
1.23 \\
0.74 \\
1.28 \\
1.44\end{array}$ \\
\hline Crystal & $\begin{array}{l}6670 . \\
6680 . \\
6690 . \\
6720 . \\
6710 . \\
6730 . \\
6750 . \\
6769 .\end{array}$ & $\begin{array}{r}0.00 \\
1405.00 \\
2950.00 \\
4650.00 \\
6525.54 \\
10870.54 \\
16115.00 \\
22044.00\end{array}$ & $\begin{array}{c}134.00 \\
147.00 \\
162.00 \\
178.00 \\
196.00 \\
237.70 \\
287.00 \\
\text { b }\end{array}$ & $\begin{array}{l}0.65 \\
0.75 \\
0.80 \\
0.95 \\
1.03 \\
1.23 \\
1.40 \\
. \mathrm{b}\end{array}$ \\
\hline
\end{tabular}

a Reservoir storage $\left(S_{E}\right)$, in acre-feet for an elevation $E$, can be calculated from the following equation:

$$
\mathrm{S}_{\mathrm{E}}=\mathrm{a}+\mathrm{b}\left(\mathrm{E}-\mathrm{E}_{\mathrm{b}}\right)+\mathrm{c}\left(\mathrm{E}-\mathrm{E}_{\mathrm{b}}\right)^{2}
$$

where $E_{b}$ is the next elevation in the table that is lower than $E$; and $a, b$, and $c$ are coefficients in the table corresponding to $\mathrm{E}_{\mathrm{b}}$.

b Value missing from table provided by the Bureau of Reclamation.

Source: Bureau of Reclamation, Salt Lake City, Utah Office, April 1992; reformatted from electronic transmittal. 
TABLE 6 Daily Reservoir Operations at the Aspinall Unit, October 1982

\begin{tabular}{|c|c|c|c|c|c|c|c|}
\hline \multirow[b]{2}{*}{ Date } & \multicolumn{7}{|c|}{ Daily Operations at Blue Mesa Reservoir } \\
\hline & $\begin{array}{l}\text { Daily } \\
\text { Inflow } \\
\text { (cfs) }\end{array}$ & $\begin{array}{c}\text { Power } \\
\text { Release } \\
\text { (cfs) }\end{array}$ & $\begin{array}{l}\text { Other } \\
\text { Release } \\
\text { (cfs) }\end{array}$ & $\begin{array}{c}\text { Total } \\
\text { Release } \\
\text { (acre-feet) }\end{array}$ & $\begin{array}{c}\text { Daily } \\
\text { Evaporation } \\
\text { (cfs) }\end{array}$ & $\begin{array}{c}\text { Storage }^{a} \\
\text { (acre-feet) }\end{array}$ & $\begin{array}{l}\text { Elevation }{ }^{a} \\
\text { (ft) }\end{array}$ \\
\hline $10 / 1$ & 1,344 & 1,320 & 0 & 2,618 & 12 & 739,203 & $7,509.25$ \\
\hline $10 / 2$ & 1,233 & 1,320 & 0 & 2,618 & 12 & 739,007 & $7,609.23$ \\
\hline $10 / 3$ & 1,353 & 1,320 & 0 & 2,618 & 12 & 739,047 & $7,509.23$ \\
\hline $10 / 4$ & 1,248 & 1,320 & 0 & 2,618 & 12 & 738,881 & $7,509.22$ \\
\hline $10 / 5$ & 1,285 & 1,320 & 0 & 2,618 & 11 & 738,789 & $7,509.20$ \\
\hline $10 / 6$ & 1,145 & 1,320 & 0 & 2,618 & 11 & 738,421 & $7,509.16$ \\
\hline $10 / 7$ & 1,223 & 1,320 & 0 & 2,618 & 11 & 738,207 & $7,509.14$ \\
\hline $10 / 8$ & 1,376 & 1,320 & 0 & 2,618 & 11 & 738,297 & $7,509.15$ \\
\hline $10 / 9$ & 1,244 & 1,320 & 0 & 2,618 & 10 & 738,127 & $7,509.13$ \\
\hline $10 / 10$ & 1,091 & 1,320 & 0 & 2,618 & 10 & 737,654 & $7,509.07$ \\
\hline $10 / 11$ & 1,335 & 1,320 & 0 & 2,618 & 10 & 737,663 & $7,509.07$ \\
\hline $10 / 12$ & 1,240 & 1,320 & 0 & 2,618 & 10 & 737,485 & $7,509.05$ \\
\hline $10 / 13$ & 1,172 & 1,320 & 0 & 2,618 & 9 & 737,174 & $7,509.02$ \\
\hline $10 / 14$ & 1,023 & 1,320 & 0 & 2,618 & 9 & 736,567 & $7,508.95$ \\
\hline $10 / 15$ & 1,209 & 1,320 & 0 & 2,618 & 9 & 736,329 & $7,508.92$ \\
\hline $10 / 16$ & 1,089 & 1,320 & 0 & 2,618 & 9 & 735,853 & $7,508.86$ \\
\hline $10 / 17$ & 1,290 & 1,320 & 0 & 2,618 & 8 & 735,778 & $7,508.86$ \\
\hline $10 / 18$ & 1,036 & 1,320 & 0 & 2,618 & 8 & 735,198 & $7,508.79$ \\
\hline $10 / 19$ & 1,047 & 1,320 & 0 & 2,618 & 8 & 734,640 & $7,508.72$ \\
\hline $10 / 20$ & 892 & 1,320 & 0 & 2,618 & 8 & 733,776 & $7,508.62$ \\
\hline $10 / 21$ & 1,006 & 1,320 & 0 & 2,618 & 8 & 733,138 & $7,508.55$ \\
\hline $10 / 22$ & 1,016 & 1,320 & 0 & 2,618 & 8 & 732,520 & $7,508.48$ \\
\hline $10 / 23$ & 901 & 1,320 & 0 & 2,618 & 7 & 731,674 & $7,508.38$ \\
\hline $10 / 24$ & 742 & 1,320 & 0 & 2,618 & 7 & 730,513 & $7,508.24$ \\
\hline $10 / 25$ & 789 & 1,320 & 0 & 2,618 & 7 & 729,446 & $7,508.12$ \\
\hline $10 / 26$ & 701 & 1,320 & 0 & 2,618 & 7 & 728,205 & $7,507.98$ \\
\hline $10 / 27$ & 630 & 1,320 & 0 & 2,618 & 7 & 726,823 & $7,507.82$ \\
\hline $10 / 28$ & 727 & 1,320 & 0 & 2,618 & 7 & 725,632 & $7,507.68$ \\
\hline $10 / 29$ & 723 & 1,320 & 0 & 2,618 & 7 & 724,435 & $7,507.54$ \\
\hline $10 / 30$ & 584 & 1,320 & 0 & 2,618 & 6 & 722,963 & $7,507.36$ \\
\hline $10 / 31$ & 740 & 1,320 & 0 & 2,618 & 6 & 721,800 & $7,507.23$ \\
\hline $\begin{array}{l}\text { Monthly total } \\
\text { (acre-feet) }\end{array}$ & 64,331 & & & & & & \\
\hline
\end{tabular}


TABLE 6 (Cont.)

\begin{tabular}{|c|c|c|c|c|c|c|c|c|c|c|}
\hline \multirow[b]{2}{*}{ Date } & \multicolumn{10}{|c|}{ Daily Operations at Morrow Point Reservoir } \\
\hline & $\begin{array}{c}\text { Inflow } \\
\text { from } \\
\text { Blue Mesa } \\
\text { (cfs) }\end{array}$ & $\begin{array}{c}\text { Side } \\
\text { Inflow } \\
\text { (cfs) }\end{array}$ & $\begin{array}{c}\text { Total } \\
\text { Inflow } \\
(\mathrm{cfg})\end{array}$ & $\begin{array}{l}\text { Power } \\
\text { Release } \\
\text { (cfs) }\end{array}$ & $\begin{array}{l}\text { Other } \\
\text { Release } \\
\text { (cfs) }\end{array}$ & $\begin{array}{c}\text { Total } \\
\text { Release } \\
\text { (acre-feet) }\end{array}$ & $\begin{array}{c}\text { Daily } \\
\text { Evaporation } \\
\text { (cfs) }\end{array}$ & $\begin{array}{l}\text { Storage }^{b} \\
\text { (acre-feet) }\end{array}$ & $\begin{array}{c}\text { Elevation }{ }^{\mathrm{b}} \\
\text { (ft) }\end{array}$ & $\begin{array}{c}\text { Elevation } \\
\text { Difference } \\
(\mathrm{ft})\end{array}$ \\
\hline $10 / 1$ & 1,320 & 77 & 1,397 & 1,410 & 0 & 2,797 & 0 & 112,742 & $7,154.44$ & 0.03 \\
\hline $10 / 2$ & 1,320 & 108 & 1,428 & 1,410 & 0 & 2,797 & 0 & 112,779 & $7,154.48$ & 0.05 \\
\hline $10 / 3$ & 1,320 & 42 & 1,362 & 1,410 & 0 & 2,797 & 0 & 112,683 & $7,154.36$ & 0.12 \\
\hline $10 / 4$ & 1,320 & 90 & 1,410 & 1,410 & 0 & 2,797 & 0 & 112,683 & $7,154.36$ & 0.00 \\
\hline $10 / 5$ & 1,320 & 70 & 1,390 & 1,410 & 0 & 2,797 & 0 & 112,644 & $7,154.32$ & 0.05 \\
\hline $10 / 6$ & 1,320 & 95 & 1,415 & 1,410 & 0 & 2,797 & 0 & 112,654 & $7,154.33$ & 0.01 \\
\hline $10 / 7$ & 1,320 & 79 & 1,399 & 1,410 & 0 & 2,797 & 0 & 112,632 & $7,154.30$ & 0.03 \\
\hline $10 / 8$ & 1,320 & 64 & 1,384 & 1,410 & 0 & 2,797 & 0 & 112,581 & $7,154.24$ & 0.06 \\
\hline $10 / 9$ & 1,320 & 106 & 1,426 & 1,410 & 0 & 2,797 & 0 & 112,613 & $7,154,28$ & 0.04 \\
\hline $10 / 10$ & 1,320 & 71 & 1,391 & 1,410 & 0 & 2,797 & 0 & 112,576 & $7,154.23$ & 0.05 \\
\hline $10 / 11$ & 1,320 & 85 & 1,405 & 1,410 & 0 & 2,797 & 0 & 112,566 & $7,154.22$ & 0.01 \\
\hline $10 / 12$ & 1,320 & 68 & 1,388 & 1,410 & 0 & 2,797 & 0 & 112,522 & $7,154.16$ & 0.06 \\
\hline $10 / 13$ & 1,320 & 92 & 1,412 & 1,410 & 0 & 2,797 & 0 & 112,525 & $7,154.17$ & 0.00 \\
\hline $10 / 14$ & 1,320 & 104 & 1,424 & 1,410 & 0 & 2,797 & 0 & 112,553 & $7,154.20$ & 0.04 \\
\hline $10 / 15$ & 1,320 & 89 & 1,409 & 1,410 & 0 & 2,797 & 0 & 112,552 & $7,154.20$ & 0.00 \\
\hline $10 / 16$ & 1,320 & 89 & 1,409 & 1,410 & 0 & 2,797 & 0 & 112,550 & $7,154.20$ & 0.00 \\
\hline $10 / 17$ & 1,320 & 66 & 1,386 & 1,410 & 0 & 2,797 & 0 & 112,501 & $7,154.14$ & 0.06 \\
\hline $10 / 18$ & 1,320 & 87 & 1,407 & 1,410 & 0 & 2,797 & 0 & 112,495 & $7,154.13$ & 0.01 \\
\hline $10 / 19$ & 1,320 & 79 & 1,399 & 1,410 & 0 & 2,797 & 0 & 112,473 & $7,154.10$ & 0.03 \\
\hline $10 / 20$ & 1,320 & 65 & 1,385 & 1,410 & 0 & 2,797 & 0 & 112,424 & $7,154.04$ & 0.06 \\
\hline $10 / 21$ & 1,320 & 39 & 1,359 & 1,410 & 0 & 2,797 & 0 & 112,323 & $7,153.91$ & 0.13 \\
\hline $10 / 22$ & 1,320 & 76 & 1,396 & 1,410 & 0 & 2,797 & 0 & 112,296 & $7,153.88$ & 0.03 \\
\hline $10 / 23$ & 1,320 & 72 & 1,392 & 1,410 & 0 & 2,797 & 0 & 112,261 & $7,153.83$ & 0.04 \\
\hline $10 / 24$ & 1,320 & 62 & 1,382 & 1,410 & 0 & 2,797 & 0 & 112,205 & $7,153.76$ & 0.07 \\
\hline $10 / 25$ & 1,320 & 84 & 1,404 & 1,410 & 0 & 2,797 & 0 & 112,192 & $7,153.75$ & 0.02 \\
\hline $10 / 26$ & 1,320 & 90 & 1,410 & 1,410 & 0 & 2,797 & 0 & 112,193 & $7,153.75$ & 0.00 \\
\hline $10 / 27$ & 1,320 & 119 & 1,439 & 1,410 & 0 & 2,797 & 0 & 112,251 & $7,153.82$ & 0.07 \\
\hline $10 / 28$ & 1,320 & 86 & 1,406 & 1,410 & 0 & 2,797 & 0 & 112,244 & $7,153.81$ & 0.01 \\
\hline $10 / 29$ & 1,320 & 76 & 1,396 & 1,410 & 0 & 2,797 & 0 & 112,217 & $7,153.78$ & 0.03 \\
\hline $10 / 30$ & 1,320 & 100 & 1,420 & 1,410 & 0 & 2,797 & 0 & 112,237 & $7,153.80$ & 0.03 \\
\hline $10 / 31$ & 1,320 & 79 & 1,399 & 1,410 & 0 & 2,797 & 0 & 112,216 & $7,153.78$ & 0.03 \\
\hline $\begin{array}{l}\text { Monthly total } \\
\text { (acre-feet) }\end{array}$ & . & & 86,143 & & & & & & & \\
\hline
\end{tabular}


TABLE 6 (Cont.)

\begin{tabular}{|c|c|c|c|c|c|c|c|c|c|c|c|c|c|}
\hline \multirow[b]{3}{*}{ Dato } & \multirow{3}{*}{$\begin{array}{c}\text { Inflow } \\
\text { from } \\
\text { Morrow } \\
\text { Point } \\
\text { (cfe) }\end{array}$} & \multirow{3}{*}{$\begin{array}{c}\text { Side } \\
\text { Inflow } \\
(\mathrm{cfs})\end{array}$} & \multirow{3}{*}{$\begin{array}{l}\text { Total } \\
\text { Inflow } \\
\text { (cfs) }\end{array}$} & \multirow{3}{*}{$\begin{array}{c}\text { Power } \\
\text { Release } \\
\text { (cfs) }\end{array}$} & \multirow{3}{*}{$\begin{array}{l}\text { Other } \\
\text { Release } \\
\text { (cfs) }\end{array}$} & \multicolumn{4}{|c|}{ Daily Operations at Crystal Reservoir } & \multirow{3}{*}{$\begin{array}{c}\text { Elevation } \\
\text { Difference } \\
\text { (ft) }\end{array}$} & & & \multirow{3}{*}{$\begin{array}{c}\text { Low } \\
\text { Eleva- } \\
\text { tion } \\
\text { Test } \\
\text { (ft) }\end{array}$} \\
\hline & & & & & & Total & $\begin{array}{l}\text { Daily } \\
\text { Evapo }\end{array}$ & & & & \multicolumn{2}{|c|}{ Fluctuation Test (ft) } & \\
\hline & & & & & & (acre-feet) & (cfs) & (acre-feet) & (ft) & & 24-hour & 48-hour & \\
\hline $10 / 1$ & 1,410 & 172 & 1,582 & 1,600 & 0 & 3,173 & 0 & 16,068 & $6,749.84$ & 0.12 & 0.12 & - & -0.12 \\
\hline $10 / 2$ & 1,410 & 241 & 1,651 & 1,600 & 0 & 3,173 & 0 & 16,169 & $6,750.19$ & 0.95 & 0.95 & 0.35 & 0.35 \\
\hline $10 / 3$ & 1,410 & 93 & 1,503 & 1,600 & 0 & 3,173 & 0 & 15,977 & $6,749.52$ & 0.67 & 0.67 & 0.67 & -0.67 \\
\hline $10 / 4$ & 1,410 & 200 & 1,610 & 1,600 & 0 & 3,173 & 0 & 15,997 & $6,749.59$ & 0.07 & 0.07 & 0.67 & 0.07 \\
\hline $10 / 5$ & 1,410 & 157 & 1,567 & 1,600 & 0 & 3,173 & 0 & 15,931 & $6,749.36$ & 0.23 & 0.23 & 0.23 & -0.23 \\
\hline $10 / 6$ & 1,410 & 212 & 1,622 & 1,600 & 0 & 3,173 & 0 & 15,975 & $6,749.51$ & 0.15 & 0.15 & 0.23 & 0.15 \\
\hline $10 / 7$ & 1,410 & 175 & 1,685 & 1,600 & 0 & 3,173 & 0 & 15,946 & $6,749.41$ & 0.10 & 0.10 & 0.15 & -0.10 \\
\hline $10 / 8$ & 1,410 & 142 & 1,652 & 1,600 & 0 & 3,173 & 0 & 15,851 & $6,749.08$ & 0.33 & 0.33 & 0.43 & .0 .33 \\
\hline $10 / 9$ & 1,410 & 237 & 1,647 & 1,600 & 0 & 3,173 & 0 & 15,944 & $6,749.40$ & 0.33 & 0.39 & 0.33 & 0.33 \\
\hline $10 / 10$ & 1,410 & 159 & 1,669 & 1,600 & 0 & 3,173 & 0 & 15,882 & $6,749.19$ & 0.22 & 0.22 & 0.33 & -0.22 \\
\hline $10 / 11$ & 1,410 & 189 & 1,699 & 1,600 & 0 & 3,173 & 0 & 15,880 & $6,749.18$ & 0.01 & 0.01 & 0.22 & -0.01 \\
\hline $10 / 12$ & 1,410 & 151 & 1,661 & 1,600 & 0 & 3,173 & 0 & 15,802 & $6,748.91$ & 0.27 & 0.27 & 0.28 & -0.27 \\
\hline $10 / 13$ & 1,410 & 204 & 1,614 & 1,600 & 0 & 3,173 & 0 & 15,830 & $6,749.00$ & 0.10 & 0.10 & 0.27 & 0.10 \\
\hline $10 / 14$ & 1,410 & 232 & 1,642 & 1,600 & 0 & 3,173 & 0 & 16,913 & $6,749.30$ & 0.29 & 0.29 & 0.39 & 0.29 \\
\hline $10 / 15$ & 1,410 & 199 & 1,609 & 1,600 & 0 & 8,173 & 0 & 15,990 & $6,749.36$ & 0.06 & 0.06 & 0.35 & 0.06 \\
\hline $10 / 16$ & 1,410 & 198 & 1,608 & 1,600 & 0 & 3,173 & 0 & 15,947 & $6,749.41$ & 0.06 & 0.06 & 0.12 & 0.06 \\
\hline $10 / 17$ & 1,410 & 146 & 1,656 & 1,600 & 0 & 3,173 & 0 & 15,859 & $6,749.11$ & 0.31 & 0.31 & 0.31 & -0.31 \\
\hline $10 / 18$ & 1,410 & 193 & 1,603 & 1,600 & 0 & 3,173 & 0 & 15,866 & $6,749.13$ & 0.02 & 0.02 & 0.31 & 0.02 \\
\hline $10 / 19$ & 1,410 & 176 & 1,586 & 1,600 & 0 & 3,173 & 0 & 15,838 & $6,749.03$ & 0.10 & 0.10 & 0.10 & -0.10 \\
\hline $10 / 20$ & 1,410 & 144 & 1,554 & 1,600 & 0 & 3,173 & 0 & 15,747 & $6,748.71$ & 0.32 & 0.32 & 0.42 & -0.32 \\
\hline $10 / 21$ & 1,410 & 88 & 1,498 & 1,600 & 0 & 3,173 & 0 & 15,544 & $6,747.99$ & 0.72 & 0.72 & 1.04 & -0.72 \\
\hline $10 / 22$ & 1,410 & 170 & 1,580 & 1,600 & 0 & 3,173 & 0 & 16,605 & $6,747.86$ & 0.14 & 0.14 & 0.86 & -0.14 \\
\hline $10 / 23$ & 1,410 & 161 & 1,671 & 1,600 & 0 & 3,173 & 0 & 15,447 & $6,747.65$ & 0.21 & 0.21 & 0.36 & -0.21 \\
\hline $10 / 24$ & 1,410 & 137 & 1,547 & 1,600 & 0 & 3,173 & 0 & 15,342 & $6,747.27$ & 0.37 & 0.37 & 0.68 & -0.37 \\
\hline $10 / 25$ & 1,410 & 187 & 1,697 & 1,600 & 0 & 3,173 & 0 & 15,395 & $6,747.25$ & 0.02 & 0.02 & 0.40 & -0.02 \\
\hline $10 / 26$ & 1,410 & 201 & 1,611 & 1,600 & 0 & 3,173 & 0 & 15,358 & $6,747.33$ & 0.08 & 0.08 & 0.08 & 0.08 \\
\hline $10 / 27$ & 1,410 & 266 & 1,676 & 1,600 & 0 & 3,173 & 0 & 15,508 & $6,747.86$ & 0.63 & 0.53 & 0.61 & 0.53 \\
\hline $10 / 28$ & 1,410 & 192 & 1,602 & 1,600 & 0 & 3,173 & 0 & 15,512 & $6,747.88$ & 0.01 & 0.01 & 0.55 & 0.01 \\
\hline $10 / 29$ & 1,410 & 170 & 1,580 & 1,600 & 0 & 3,173 & 0 & 15,479 & $6,747.74$ & 0.14 & 0.14 & 0.14 & -0.14 \\
\hline $10 / 30$ & 1,410 & 223 & 1,693 & 1,600 & 0 & 3,173 & 0 & 15,637 & $6,747.97$ & 0.23 & 0.23 & 0.23 & 0.23 \\
\hline $10 / 31$ & 1,410 & 177 & 1,587 & 1,600 & 0 & 3,173 & 0 & 15,511 & $6,747.88$ & 0.09 & 0.09 & 0.23 & -0.09 \\
\hline $\begin{array}{l}\text { Monthly total } \\
\text { (acre-feet) }\end{array}$ & & & 97,785 & 98,377 & 0 & & & & & & & & \\
\hline
\end{tabular}

a Initial storage $=739,179$ acre-feet; initial elevation $=7,509.25$.

b Initial storage $=112,767$ acre-feet; initial elevation $=7,154.47$ acre-feet.

c Initial storage $=16,103$ acre-feet; initial elevation $=6,749.96$. 
TABLE 7 Adjusted Daily Releases at Crystal Reservoir, April through July 1983

\begin{tabular}{|c|c|c|c|c|}
\hline \multirow[b]{2}{*}{ Day } & \multicolumn{4}{|c|}{ Daily Release (cfs) } \\
\hline & April & May & June & July \\
\hline 1 & 3,500 & 3,000 & 5,100 & 2,500 \\
\hline $\begin{array}{l}1 \\
2\end{array}$ & 3,500 & 3,000 & 5,100 & 2,500 \\
\hline 3 & 3,500 & 3,000 & 5,100 & 2,500 \\
\hline 4 & 3,500 & 3,000 & 3,600 & 2,500 \\
\hline 5 & 3,500 & 3,000 & 3,600 & 2,500 \\
\hline 6 & 3,500 & 3,000 & 3,600 & 2,500 \\
\hline 7 & 3,500 & 3,000 & 3,600 & 2,500 \\
\hline 8 & 3,500 & 3,000 & 3,600 & 2,900 \\
\hline 9 & 3,500 & 3,500 & 3,600 & 2,900 \\
\hline 10 & 3,500 & 3,500 & 3,600 & 2,900 \\
\hline 11 & 3,500 & 3,500 & 3,600 & 2,500 \\
\hline 12 & 3,400 & 3,500 & 3,600 & 2,500 \\
\hline 13 & 3,400 & 3,200 & 3,600 & 2,500 \\
\hline 14 & 3,400 & 3,200 & 4,100 & 2,500 \\
\hline 15 & 3,400 & 3,200 & 4,100 & 2,500 \\
\hline 16 & 3,400 & 3,200 & 4,100 & 2,500 \\
\hline 17 & 3,400 & 3,200 & 4,600 & 2,500 \\
\hline 18 & 3,400 & 3,200 & 4,600 & 2,500 \\
\hline 19 & 3,500 & 3,500 & 4,600 & 2,500 \\
\hline 20 & 3,500 & 3,500 & 4,600 & 2,500 \\
\hline 21 & 3,500 & 3,500 & 4,600 & 2,500 \\
\hline 22 & 3,500 & 3,800 & 4,600 & 2,500 \\
\hline 23 & 3,500 & 3,800 & 4,600 & 2,500 \\
\hline 24 & 3,600 & 3,800 & 4,600 & 2,500 \\
\hline 25 & 3,600 & 3,800 & 4,600 & 2,500 \\
\hline 26 & 3,600 & 3,800 & 4,600 & 2,500 \\
\hline 27 & 3,600 & 3,800 & 4,600 & 2,100 \\
\hline 28 & 3,600 & 3,500 & 4,600 & 2,100 \\
\hline 29 & 3,600 & 3,500 & 4,600 & 2,100 \\
\hline 30 & 3,600 & 3,500 & 4,600 & 2,100 \\
\hline 31 & & 3,500 & & 2,100 \\
\hline Average & 3,500 & 3,500 & 4,600 & 2,500 \\
\hline
\end{tabular}


TABLE 8 Maximum Allowable Fluctuations for Reservoir Releases at Morrow Point

\begin{tabular}{lccc}
\hline & \multicolumn{3}{c}{$\begin{array}{c}\text { Maximum Fluctuation as Percent of } \\
\text { 5,300-cfs Power Plant Capacity }\end{array}$} \\
\cline { 2 - 4 } Month & Moderate Year & Dry Year & Wet Year \\
\hline & & & \\
April & 40 & 40 & 40 \\
May & 30 & 40 & 30 \\
June & 25 & 40 & 30 \\
July & 40 & 40 & 30 \\
\hline
\end{tabular}


TABLE 9 Daily Release Patterns for Blue Mesa Reservoir ${ }^{\mathrm{a}}$

\begin{tabular}{|c|c|c|c|c|c|c|c|c|c|c|c|c|}
\hline \multirow[b]{3}{*}{ Month } & \multicolumn{4}{|c|}{ Release Patterns for Moderate Year } & \multicolumn{4}{|c|}{ Release Patterns for Dry Year } & \multicolumn{4}{|c|}{ Release Patterns for Wet Year } \\
\hline & \multicolumn{3}{|c|}{$\begin{array}{l}\text { Seasonally Adjusted } \\
\text { High Fluctuating Flows }\end{array}$} & \multirow{2}{*}{$\begin{array}{l}\text { Seasonally } \\
\text { Adjusted } \\
\text { Steady } \\
\text { Flows } \\
\text { (cfs) }\end{array}$} & \multicolumn{3}{|c|}{$\begin{array}{l}\text { Seasonally Adjusted } \\
\text { High Fluctuating Flows }\end{array}$} & \multirow{2}{*}{$\begin{array}{l}\text { Seasonally } \\
\text { Adjusted } \\
\text { Steady } \\
\text { Flows } \\
\text { (cfs) }\end{array}$} & \multicolumn{3}{|c|}{$\begin{array}{l}\text { Seasonally Adjusted } \\
\text { High Fluctuating Flows }\end{array}$} & \multirow{2}{*}{$\begin{array}{l}\text { Seasonally } \\
\text { Adjusted } \\
\text { Steady } \\
\text { Flows } \\
\text { (cfs) }\end{array}$} \\
\hline & $\begin{array}{l}\text { Min. } \\
\text { Release } \\
\text { (cfs) }\end{array}$ & $\begin{array}{l}\text { Max. } \\
\text { Release }^{b} \\
\text { (cfs) }\end{array}$ & $\begin{array}{c}\text { On-Peak } \\
\text { Duration } \\
\text { (hours) }\end{array}$ & & $\begin{array}{l}\text { Min. } \\
\text { Release } \\
\text { (cfs) }\end{array}$ & $\begin{array}{l}\text { Max. } \\
\text { Release }^{b} \\
\text { (cfs) }\end{array}$ & $\begin{array}{c}\text { On-Peak } \\
\text { Duration } \\
\text { (hours) }\end{array}$ & & $\begin{array}{l}\text { Min. } \\
\text { Release } \\
\text { (cfs) }\end{array}$ & $\begin{array}{l}\text { Max. } \\
\text { Release }^{b} \\
\text { (cfs) }\end{array}$ & $\begin{array}{c}\text { On-Peak } \\
\text { Duration } \\
\text { (hours) }\end{array}$ & \\
\hline Oct & 0 & 3,700 & 9 & 1,570 & 0 & 3,700 & 3 & 650 & 0 & 3,700 & 7 & 1,320 \\
\hline Nov & 0 & 3,700 & 6 & 1,200 & 0 & 3,700 & $<1$ & 180 & 0 & 3,700 & 8 & 1,400 \\
\hline Dec & 0 & 3,700 & 5 & 1,050 & 0 & 3,600 & $<1$ & 150 & 0 & 3,700 & 8 & 1,500 \\
\hline Jan & 0 & 3,700 & 2 & 500 & 0 & 3,700 & $<1$ & 200 & 0 & 3,700 & 8 & 1,540 \\
\hline $\mathrm{Feb}$ & 0 & 3,700 & 2 & 510 & 0 & 3,700 & $<1$ & 250 & 0 & 3,700 & 8 & 1,470 \\
\hline Mar & 0 & 3,700 & 2 & 500 & 0 & 3,700 & $<1$ & 200 & 0 & 3,700 & 8 & 1,450 \\
\hline Apr & 0 & 3,700 & 9 & 1,600 & 0 & 3,700 & 3 & 760 & 2,260 & 3,700 & 15 & 3,220 \\
\hline May & 0 & 3,700 & 14 & 2,370 & 0 & 3,700 & 4 & 900 & 0 & 3,700 & 13 & 2,200 \\
\hline Jun & 1,750 & 3,700 & 15 & 3,050 & 0 & 3,700 & 6 & 1,150 & 0 & 3,700 & 14 & 2,330 \\
\hline Jul & 0 & 3,700 & 14 & 2,350 & 0 & 3,700 & 7 & 1,300 & 0 & 3,700 & 9 & 1,550 \\
\hline Aug & 0 & 3,700 & 10 & 1,750 & 0 & 3,700 & 7 & 1,300 & 0 & 3,700 & 6 & 1,100 \\
\hline Sep & 0 & 3,700 & 10 & 1,750 & 0 & 3,700 & 7 & 1,300 & 0 & 3,700 & 8 & 1,500 \\
\hline
\end{tabular}

a The average annual releases would be $1,520 \mathrm{cfs}$ for the moderate year, $1,710 \mathrm{cfs}$ for the wet year, and $697 \mathrm{cfs}$ for the dry year.

b Maximum release of $3,700 \mathrm{cfs}$ assumes full reservoir condition. 
TABLE 10 Daily Release Patterns for Morrow Point Reservoir ${ }^{a}$

\begin{tabular}{|c|c|c|c|c|c|c|c|c|c|c|c|c|}
\hline \multirow[b]{3}{*}{ Month } & \multicolumn{4}{|c|}{ Release Patterns for Moderate Year } & \multicolumn{4}{|c|}{ Release Patterns for Dry Year } & \multicolumn{4}{|c|}{ Release Patterns for Wet Year } \\
\hline & \multicolumn{3}{|c|}{$\begin{array}{l}\text { Seasonally Adjusted } \\
\text { High Fluctuating Flows }\end{array}$} & \multirow{2}{*}{$\begin{array}{l}\text { Seasonally } \\
\text { Adjusted } \\
\text { Steady } \\
\text { Flows } \\
\text { (cfs) }\end{array}$} & \multicolumn{3}{|c|}{$\begin{array}{l}\text { Seasonally Adjusted } \\
\text { High Fluctuating Flows }\end{array}$} & \multirow{2}{*}{$\begin{array}{c}\text { Seasonally } \\
\text { Adjusted } \\
\text { Steady } \\
\text { Flows } \\
\text { (cfs) }\end{array}$} & \multicolumn{3}{|c|}{$\begin{array}{c}\text { Seasonally Adjusted } \\
\text { High Fluctuating Flows }\end{array}$} & \multirow{2}{*}{$\begin{array}{c}\text { Seasonally } \\
\text { Adjusted } \\
\text { Steady } \\
\text { Flows } \\
\text { (cfs) }\end{array}$} \\
\hline & $\begin{array}{c}\text { Min. } \\
\text { Release } \\
\text { (cfs) }\end{array}$ & $\begin{array}{c}\text { Max. } \\
\text { Release }^{b} \\
\text { (cfs) }\end{array}$ & $\begin{array}{c}\text { On-Peak } \\
\text { Duration } \\
\text { (hours) }\end{array}$ & & $\begin{array}{c}\text { Min. } \\
\text { Release } \\
\text { (cfs) }\end{array}$ & $\begin{array}{c}\text { Max. } \\
\text { Release }^{\mathrm{b}} \\
\text { (cfs) }\end{array}$ & $\begin{array}{c}\text { On-Peak } \\
\text { Duration } \\
\text { (hours) }\end{array}$ & & $\begin{array}{c}\text { Min. } \\
\text { Release } \\
\text { (cfs) }\end{array}$ & $\begin{array}{l}\text { Max. } \\
\text { Release }^{b} \\
\text { (cfs) }\end{array}$ & $\begin{array}{c}\text { On-Peak } \\
\text { Duration } \\
\text { (hours) }\end{array}$ & \\
\hline Oct & 0 & 5,300 & 6 & 1,700 & 0 & 5,300 & 2 & 720 & 0 & 5,300 & $\mathbf{5}$ & 1,410 \\
\hline Nov & 0 & 5,300 & 4 & 1,280 & 0 & 4,800 & $<1$ & 200 & 0 & 5,300 & 5 & 1,510 \\
\hline Dec & 0 & 5,300 & 3 & 1,100 & 0 & 4,800 & $<1$ & 200 & 0 & 5,300 & 5 & 1,520 \\
\hline Jan & 0 & 5,300 & 1 & 570 & 0 & 5,270 & $<1$ & 220 & 0 & 5,300 & 5 & 1,620 \\
\hline Feb & 0 & 5,300 & 1 & 580 & 0 & 5,300 & $<1$ & 270 & 0 & 5,300 & 5 & 1,540 \\
\hline Mar & 0 & 5,300 & 1 & 650 & 0 & 5,300 & $<1$ & 260 & 0 & 5,300 & 5 & 1,540 \\
\hline Apr & 557 & 2,680 & 15 & 1,970 & 0 & 2,120 & 9 & 970 & 1,930 & 4,050 & 15 & 3,340 \\
\hline May & 1,830 & 3,420 & 15 & 2,890 & 0 & 2,120 & 11 & 1,090 & 1,700 & 3,300 & 15 & 2,760 \\
\hline Jun & 2,440 & 3,770 & 15 & 3,320 & 0 & 2,120 & 12 & 1,220 & 2,120 & 3,710 & 15 & 3,180 \\
\hline Jul & 1,070 & 3,190 & 17 & 2,480 & 0 & 2,120 & 14 & 1,350 & 780 & 2,370 & 15 & 1,840 \\
\hline Aug & 0 & 5,300 & 7 & 1,770 & 0 & 5,300 & 5 & 1,400 & 0 & 5,300 & 4 & 1,300 \\
\hline Sep & 0 & 5,300 & 7 & 1,820 & 0 & 5,300 & 4 & 1,300 & 0 & 5,300 & 5 & 1,510 \\
\hline
\end{tabular}

a The annual average releases would be $1,680 \mathrm{cfs}$ for the moderate year, $1,910 \mathrm{cfs}$ for the wet year, and $769 \mathrm{cfs}$ for the dry year.

b Maximum release of $5,300 \mathrm{cfs}$ assumes full reservoir condition. 
TABLE 11 Maximum Daily Reservoir Surface Fluctuations under the Seasonally Adjusted High Fluctuating Flow Scenario during a Moderate Year

\begin{tabular}{|c|c|c|c|c|c|c|}
\hline \multirow[b]{2}{*}{ Month } & \multicolumn{2}{|c|}{ Blue Mesa } & \multicolumn{2}{|c|}{ Morrow Point } & \multicolumn{2}{|c|}{ Crystal } \\
\hline & $\begin{array}{l}\text { Maximum } \\
\text { Daily } \\
\text { Fluctuation } \\
\text { (ft) }\end{array}$ & $\begin{array}{l}\% \text { Due to } \\
\text { Hourly } \\
\text { Fluctuation }\end{array}$ & $\begin{array}{l}\text { Maximum } \\
\text { Daily } \\
\text { Fluctuation } \\
\text { (ft) }\end{array}$ & $\begin{array}{l}\text { \% Due to } \\
\text { Hourly } \\
\text { Fluctuation }\end{array}$ & $\begin{array}{l}\text { Maximum } \\
\text { Daily } \\
\text { Fluctuation } \\
\text { (ft) }\end{array}$ & $\begin{array}{l}\text { \% Due to } \\
\text { Hourly } \\
\text { Fluctuation }\end{array}$ \\
\hline Oct & 0.3 & 48 & 1.1 & 92 & 8.0 & 99 \\
\hline Nov & 0.2 & 61 & 0.8 & 94 & 6.9 & 99 \\
\hline Dec & 0.2 & 39 & 0.8 & 94 & 6.4 & 96 \\
\hline Jan & 0.1 & 91 & 0.3 & 94 & 4.0 & 96 \\
\hline Feb & 0.1 & 100 & 0.3 & 91 & 3.8 & 100 \\
\hline Mar & 0.1 & 29 & 0.4 & 70 & 4.3 & 92 \\
\hline $\mathrm{Apr}$ & 0.2 & 41 & 1.5 & 99 & 3.2 & 98 \\
\hline May & 0.5 & 4 & 1.3 & 98 & 2.4 & 97 \\
\hline Jun & 0.3 & 4 & 0.4 & 88 & 2.1 & 100 \\
\hline Jul & 0.3 & 38 & 0.9 & 80 & 3.4 & 98 \\
\hline Aug & 0.3 & 41 & 1.3 & 93 & 8.1 & 98 \\
\hline Sep & 0.3 & 25 & 1.3 & 95 & 8.4 & 100 \\
\hline
\end{tabular}


TABLE 12 Maximum and Minimum Reservoir Surface Elevations on the First Day of the Month under the Seasonally Adjusted High Fluctuating Flow Scenario at the Aspinall Unit during a Moderate Year

\begin{tabular}{|c|c|c|c|c|c|c|c|c|c|c|c|c|}
\hline Reservoir/Parameter & Oct & Nov & Dec & Jan & Feb & Mar & Apr & May & Jun & Jul & Aug & Sep \\
\hline \multicolumn{13}{|l|}{ Blue Mesa } \\
\hline $\begin{array}{l}\text { Starting storage } \\
\text { (acre-feet) }\end{array}$ & 660,000 & 625,462 & 605,086 & 670,920 & 567,864 & 568,124 & 592,603 & 622,739 & 747,592 & 820,553 & 771,736 & 727,623 \\
\hline Inflow (cfB) & 1,017 & 862 & 497 & 452 & 517 & 903 & 2,114 & 4,415 & 4,299 & 1,581 & 1,051 & 745 \\
\hline \multicolumn{13}{|l|}{$\begin{array}{l}\text { End-of-hour storage } \\
\text { (acre-feet) }\end{array}$} \\
\hline 1:00 a.m. & 660,084 & 625,533 & 605,127 & 570,957 & 567,907 & 568,199 & 692,778 & 623,103 & 747,803 & 820,684 & 771,823 & 727,685 \\
\hline 2:00 a.m & 660,168 & 625,604 & 605,168 & 570,995 & 667,949 & 668,273 & 592,952 & 629,466 & 748,013 & 820,814 & 771,910 & 727,746 \\
\hline 3:00 a.m. & 660,252 & 625,676 & 605,209 & 571,032 & 567,992 & 568,348 & 593,127 & 623,830 & 748,224 & 820,945 & 771,997 & 727,808 \\
\hline 4:00 a.m. & 660,336 & 625,747 & 605,250 & 571,069 & 568,035 & 568,423 & 593,302 & 624,194 & 748,435 & 821,076 & 772,083 & 727,869 \\
\hline 6:00 a.m. & 660,420 & 625,818 & 605,291 & 671,107 & 568,078 & 568,497 & 599,477 & 624,557 & 748,645 & 821,206 & 772,170 & 727,931 \\
\hline 6:00 a.m. & 660,504 & 625,889 & 605,932 & 571,144 & 568,120 & 568,672 & 593,651 & 624,921 & 748,856 & 821,337 & 772,257 & 727,992 \\
\hline 7:00 a.m. & 660,588 & 625,961 & 605,374 & 571,181 & 568,163 & 568,646 & 593,826 & 625,285 & 749,067 & 821,468 & 772,344 & 728,054 \\
\hline $8: 00$ a.m. & 660,672 & 626,032 & 605,415 & 571,219 & 668,206 & 568,721 & 594,001 & 625,343 & 749,116 & 821,293 & 772,431 & 728,116 \\
\hline 9:00 a.m. & 660,756 & 626,103 & 605,456 & 571,256 & 568,249 & 568,796 & 594,176 & 625,400 & 749,166 & 821,117 & 772,618 & 728,177 \\
\hline 10:00 a.m. & 660,840 & 626,174 & 605,497 & 571,294 & 568,291 & 568,870 & 594,950 & 625,458 & 749,215 & 820,942 & 772,605 & 728,239 \\
\hline 11:00 a.m. & 660,619 & 626,246 & 605,638 & 571,331 & 568,334 & 668,945 & 694,219 & 625,516 & 749,265 & 820,767 & 772,386 & 727,994 \\
\hline 12:00 Noon & 660,397 & 626,317 & 605,679 & 671,368 & 568,377 & 569,020 & 694,088 & 625,574 & 749,314 & 820,692 & 772,167 & 727,750 \\
\hline 1:00 p.m. & 660,175 & 626,082 & 605,314 & 571,406 & 568,419 & 669,094 & 593,957 & 625,632 & 749,364 & 820,417 & 771,948 & 727,506 \\
\hline 2:00 p.m. & 659,954 & 626,848 & 605,049 & 571,443 & 568,462 & 669,169 & 593,826 & 625,690 & 749,413 & 820,242 & 771,729 & 727,262 \\
\hline 3:00 p.m. & 659,732 & 625,613 & 604,785 & 571,175 & 568,199 & 568,938 & 593,695 & 625,748 & 749,463 & 820,067 & 771,510 & 727,018 \\
\hline 4:00 p.m. & 659,510 & 625,379 & 604,620 & 570,906 & 567,936 & 568,706 & 593,564 & 625,806 & 749,512 & 819,892 & 771,291 & 726,773 \\
\hline 5:00 p.m. & 659,288 & 625,144 & 604,255 & 570,638 & 567,673 & 568,475 & 693,439 & 625,864 & 749,562 & 819,716 & 771,072 & 726,529 \\
\hline $6: 00$ p.m. & 659,067 & 624,910 & 608,991 & 570,601 & 567,622 & 568,476 & 693,302 & 625,921 & 749,611 & 819,541 & 770,853 & 726,285 \\
\hline 7:00 p.m. & 658,845 & 624,675 & 603,784 & 570,638 & 567,664 & 668,550 & 693,170 & 625,979 & 749,661 & 819,366 & 770,634 & 726,041 \\
\hline 8:00 p.m. & 668,629 & 624,607 & 603,825 & 570,675 & 567,707 & 568,625 & 693,039 & 626,037 & 749,710 & 819,191 & 770,415 & 725,797 \\
\hline 9:00 p.m. & 658,651 & 624,578 & 603,866 & 570,713 & 567,750 & 568,699 & 693,098 & 626,095 & 749,760 & 819,016 & 770,196 & 725,552 \\
\hline 10:00 p.m. & 658,735 & 624,649 & 603,907 & 570,750 & 567,792 & 668,774 & 693,273 & 626,153 & 749,809 & 818,841 & 770,176 & 725,506 \\
\hline 11:00 p.m. & 658,819 & 624,720 & 603,948 & 570,787 & 567,835 & 568,849 & 693,448 & 626,403 & 749,859 & 818,897 & 770,263 & 725,568 \\
\hline 12:00 Midnight & 658,903 & 624,792 & 603,989 & 570,825 & 567,878 & 668,923 & 593,623 & 626,766 & 760,069 & 819,028 & 770,350 & 725,630 \\
\hline \multicolumn{13}{|l|}{ Storage (acre-feet) } \\
\hline Maximum & 660,840 & 626,317 & 605,679 & 671,443 & 568,462 & 569,169 & 594,350 & 626,766 & 750,069 & 821,468 & 772,605 & 728,239 \\
\hline Minimum & 658,623 & 624,507 & 603,784 & 570,601 & 667,622 & 568,124 & 592,603 & 622,739 & 747,692 & 818,841 & 770,176 & 725,506 \\
\hline \multicolumn{13}{|l|}{ Elevation (ft) } \\
\hline Maximum & $7,499.96$ & $7,495.72$ & $7,493.12$ & $7,488.76$ & $7,488.37$ & $7,488.46$ & $7,491.69$ & $7,495.77$ & $7,510.51$ & $7,518.52$ & $7,513.08$ & $7,507.98$ \\
\hline Minimum & $7,499.69$ & $7,495.49$ & $7,492.89$ & $7,488.65$ & $7,488.26$ & $7,488.33$ & $7,491.47$ & $7,495.27$ & $7,510.23$ & $7,518.23$ & $7,512.81$ & $7,607.66$ \\
\hline Difference & 0.27 & 0.23 & 0.23 & 0.11 & 0.11 & 0.14 & 0.22 & 0.50 & 0.28 & 0.29 & 0.27 & 0.32 \\
\hline
\end{tabular}


TABLE 12 (Cont.)

\begin{tabular}{|c|c|c|c|c|c|c|c|c|c|c|c|c|}
\hline Reservoir/Parameter & Oct & Nov & Dec & Jan & Feb & Mar & Apr & May & Jun & Jul & Aug & Sep \\
\hline \multicolumn{13}{|l|}{ Morrow Point } \\
\hline $\begin{array}{l}\text { Starting storage } \\
\text { (acre-feet) }\end{array}$ & 114,833 & 112,632 & 111,489 & 112,627 & 112,061 & 112,744 & 109,528 & 109,707 & 110,439 & 111,730 . & 107,420 & 109,688 \\
\hline Side inflow (cfs) & 94 & 61 & 69 & 61 & 82 & 98 & 373 & 534 & 311 & 74 & 58 & 44 \\
\hline \multicolumn{13}{|l|}{$\begin{array}{l}\text { End-of-hour storage } \\
\text { (acre-feet) }\end{array}$} \\
\hline 1:00 a.m. & 114,841 & 112,637 & 111,495 & 112,632 & 112,068 & 112,752 & 109,513 & 109,600 & 110,408 & 111,648 & 107,425 & 109,692 \\
\hline 2:00 a.m. & 114,849 & 112,642 & 111,500 & 112,637 & 112,075 & 112,760 & 109,498 & 109,493 & 110,376 & 111,566 & 107,430 & 109,695 \\
\hline 3:00 a.m. & 114,856 & 112,647 & 111,506 & 112,642 & 112,081 & 112,768 & 109,482 & 109,386 & 110,345 & 111,484 & 107,434 & 109,699 \\
\hline 4:00 a.m. & 114,864 & 112,652 & 111,512 & 112,647 & 112,088 & 112,776 & 109,467 & 109,279 & 110,313 & 111,402 & 107,439 & 109,703 \\
\hline 5:00 a.m. & 114,872 & 112,657 & 111,518 & 112,652 & 112,095 & 112,784 & 109,452 & 109,171 & 110,282 & 111,320 & 107,444 & 109,706 \\
\hline 6:00 a.m. & 114,880 & 112,662 & 111,523 & 112,657 & 112,102 & 112,793 & 109,437 & 109,064 & 110,250 & 111,238 & 107,449 & 109,710 \\
\hline 7:00 a.m. & 114,887 & 112,667 & 111,529 & 112,662 & 112,108 & 112,801 & 109,422 & 108,957 & 110,219 & 111,166 & 107,454 & 109,713 \\
\hline $8: 00$ a.m. & 114,895 & 112,672 & 111,535 & 112,667 & 112,115 & 112,809 & 109,231 & 109,025 & 110,239 & 111,204 & 107,458 & 109,717 \\
\hline 9:00 a.m. & 114,903 & 112,677 & 111,540 & 112,672 & 112,122 & 112,817 & 109,041 & 109,092 & 110,259 & 111,253 & 107,463 & 109,721 \\
\hline 10:00 a.m. & 114,911 & 112,682 & 111,546 & 112,677 & 112,129 & 112,825 & 108,851 & 109,159 & 110,279 & 111,301 & 107,468 & 109,724 \\
\hline 11:00 a.m. & 115,224 & 112,687 & 111,552 & 112,682 & 112,136 & 112,833 & 108,966 & 109,226 & 110,300 & 111,350 & 107,779 & 110,034 \\
\hline 12:00 Noon & 115,538 & 112,692 & 111,557 & 112,687 & 112,142 & 112,841 & 109,081 & 109,294 & 110,320 & 111,398 & 107,651 & 109,905 \\
\hline 1:00 p.m. & 115,413 & 113,003 & 111,869 & 112,693 & 112,149 & 112,849 & 109,197 & 109,361 & 110,340 & 111,447 & 107,524 & 109,777 \\
\hline $2: 00$ p.m. & 115,289 & 112,876 & 111,742 & 112,698 & 112,156 & 112,857 & 109,312 & 109,428 & 110,360 & 111,496 & 107,396 & 109,648 \\
\hline 3:00 p.m. & 116,164 & 112,749 & 111,616 & 112,570 & 112,030 & 112,733 & 109,428 & 109,495 & 110,380 & 111,544 & 107,269 & 109,519 \\
\hline 4:00 p.m. & 115,040 & 112,622 & 111,489 & 112,443 & 111,905 & 112,609 & 109,543 & 109,563 & 110,401 & 111,593 & 107,141 & 109,391 \\
\hline 5:00 p.m. & 114,915 & 112,495 & 111,363 & 112,499 & 111,943 & 112,510 & 109,658 & 109,630 & 110,421 & 111,641 & 107,014 & 109,262 \\
\hline 6:00 p.m. & 114,791 & 112,367 & 111,245 & 112,579 & 112,044 & 112,592 & 109,774 & 109,697 & 110,441 & 111,690 & 106,886 & 109,134 \\
\hline 7:00 p.m. & 114,667 & 112,329 & 111,498 & 112,584 & 112,051 & 112,600 & 109,889 & 109,765 & 110,461 & 111,738 & 106,759 & 109,005 \\
\hline $8: 00$ p.m. & 114,674 & 112,674 & 111,504 & 112,589 & 112,058 & 112,608 & 110,005 & 109,832 & 110,481 & 111,787 & 107,063 & 109,209 \\
\hline 9:00 p.m. & 114,738 & 112,579 & 111,510 & 112,594 & 112,064 & 112,617 & 109,930 & 109,899 & 110,502 & 111,835 & 107,374 & 109,518 \\
\hline 10:00 p.m. & 114,746 & 112,584 & 111,615 & 112,599 & 112,071 & 112,625 & 109,740 & 109,966 & 110,522 & 111,884 & 107,486 & 109,629 \\
\hline 11:00 p.m. & 114,754 & 112,689 & 111,621 & 112,604 & 112,078 & 112,633 & 109,549 & 109,842 & 110,542 & 111,701 & 107,491 & 109,633 \\
\hline 12:00 Midnight & 114,762 & 112,594 & 111,627 & 112,609 & 112,085 & 112,641 & 109,534 & 109,735 & 110,510 & 111,619 & 107,495 & 109,636 \\
\hline \multicolumn{13}{|l|}{ Storage (acre-feet) } \\
\hline Maximum & 115,538 & 113,003 & 111,869 & 112,698 & 112,156 & 112,857 & 110,005 & 109,966 & 110,542 & 111,884 & 107,779 & 110,034 \\
\hline Minimum & 114,667 & 112,329 & 111,245 & 112,443 & 111,905 & 112,510 & 108,851 & 108,957 & 110,219 & 111,156 & 106,759 & 109,005 \\
\hline \multicolumn{13}{|l|}{ Elevation (ft) } \\
\hline Maximum & $7,167.92$ & $7,154.77$ & $7,153.34$ & $7,154.38$ & $7,153.70$ & $7,154.68$ & $7,150.97$ & $7,150.92$ & $7,151.65$ & $7,153.36$ & $7,148.10$ & $7,151.00$ \\
\hline Minimum & $7,156.84$ & $7,153.92$ & $7,152.65$ & $7,154.06$ & $7,153.38$ & $7,154.15$ & $7,149.49$ & $7,149.62$ & $7,151,24$ & $7,152.43$ & $7,146.77$ & $7,149.68$ \\
\hline Difference & 1.08 & 0.85 & 0.79 & 0.32 & 0.32 & 0.44 & 1.48 & 1.30 & 0.41 & 0.92 & 1.33 & 1.32 \\
\hline
\end{tabular}


TABLE 12 (Cont.)

\begin{tabular}{|c|c|c|c|c|c|c|c|c|c|c|c|c|}
\hline Reservoir/Parameter & Oct & Nov & Dec & Jan & Feb & Mar & Apr & May & Jun & Jul & Aug & Sep \\
\hline \multicolumn{13}{|l|}{ Crystal } \\
\hline $\begin{array}{l}\text { Starting storage } \\
\text { (acre-feet) }\end{array}$ & 15,829 & 15,276 & 14,443 & 12,413 & 13,766 & 13,822 & 16,885 & 16,418 & 15,926 & 15,926 & 16,480 & 15,311 \\
\hline Side inflow (cfs) & 210 & 135 & 152 & 135 & 123 & 147 & 275 & 680 & 607 & 164 & 130 & 97 \\
\hline Steady release (cfs) & 1,919 & 1,429 & 1,285 & 683 & 702 & 748 & 2,252 & 3,578 & 9,892 & 2,685 & 1,919 & 1,916 \\
\hline \multicolumn{13}{|l|}{$\begin{array}{l}\text { End-of-hour storage } \\
\text { (acre-feet) }\end{array}$} \\
\hline 1:00 a.m. & 15,688 & 15,169 & 14,349 & 12,368 & 13,718 & 13,772 & 16,718 & 16,330 & 15,853 & 15,810 & 16,332 & 15,161 \\
\hline 2:00 a.m. & 15,647 & 15,062 & 14,256 & 12,322 & 13,670 & 13,723 & 16,600 & 16,241 & 15,780 & 15,694 & 16,184 & 15,010 \\
\hline 3:00 a.m. & 16,405 & 14,955 & 14,162 & 12,277 & 13,622 & 13,673 & 16,483 & 16,153 & 15,707 & 15,578 & 16,036 & 14,860 \\
\hline 4:00 a.m. & 15,264 & 14,848 & 14,068 & 12,232 & 13,575 & 13,623 & 16,365 & 16,065 & 15,634 & 15,462 & 15,889 & 14,710 \\
\hline 6:00 a.m. & 15,123 & 14,741 & 13,975 & 12,187 & 13,627 & 13,574 & 16,248 & 15,977 & 15,661 & 15,346 & 15,741 & 14,559 \\
\hline 6:00 a.m. & 14,982 & 14,634 & 13,881 & 12,141 & 13,479 & 13,624 & 16,131 & 15,888 & 16,488 & 15,230 & 15,593 & 14,409 \\
\hline 7:00 a.m. & 14,840 & 14,527 & 19,788 & 12,096 & 13,431 & 13,474 & 16,013 & 15,800 & 15,415 & 15,114 & 15,445 & 14,259 \\
\hline 8:00 a.m. & 14,699 & 14,420 & 13,694 & 12,051 & 13,383 & 13,425 & 16,071 & 15,843 & 15,451 & 15,173 & 15,297 & 14,108 \\
\hline 9.00 a.m. & 14,558 & 14,314 & 13,600 & 12,005 & 13,335 & 13,375 & 16,129 & 15,886 & 15,488 & 15,232 & 15,149 & 13,958 \\
\hline 11:00 a.m. & 14,276 & 14,100 & 13,413 & 11,916 & 13,240 & 13,276 & 16,245 & 15,973 & 15,561 & 15,350 & 14,854 & 13,657 \\
\hline 12:00 Noon & 14,134 & 18,993 & 13,319 & 11,870 & 13,192 & 13,226 & 16,302 & 16,016 & 16,597 & 15,409 & 16,144 & 13,945 \\
\hline 1:00 p.m. & 14,431 & 13,886 & 13,226 & 11,824 & 13,144 & 13,176 & 16,360 & 16,059 & 15,634 & 15,468 & 15,434 & 14,233 \\
\hline 2:00 p.m. & 14,728 & 14,217 & 13,570 & 11,779 & 13,096 & 13,127 & 16,418 & 16,102 & 15,670 & 15,528 & 15,724 & 14,520 \\
\hline 3:00 p.m. & 15,024 & 14,548 & 13,914 & 12,172 & 13,486 & 13,515 & 16,476 & 16,145 & 15,707 & 15,687 & 16,014 & 14,808 \\
\hline 4:00 p.m. & 15,321 & 14,879 & 14,259 & 12,564 & 13,876 & 13,903 & 16,534 & 16,188 & 15,743 & 15,646 & 16,304 & 15,096 \\
\hline 5:00 p.m. & 15,618 & 15,210 & 14,603 & 12,774 & 14,103 & 14,267 & 16,692 & 16,232 & 15,780 & 15,705 & 16,595 & 15,383 \\
\hline 6:00 p.m. & 15,915 & 15,541 & 14,939 & 12,728 & 14,055 & 14,217 & 16,649 & 16,275 & 15,816 & 15,764 & 16,885 & 15,671 \\
\hline 7:00 p.m. & 16,212 & 15,783 & 14,846 & 12,683 & 14,007 & 14,168 & 16,707 & 16,318 & 15,853 & 15,823 & 17,175 & 15,959 \\
\hline 8:00 p.m. & 16,376 & 15,676 & 14,752 & 12,638 & 13,959 & 14,118 & 16,765 & 16,361 & 15,889 & 15,882 & 17,034 & 15,914 \\
\hline 9:00 p.m. & 16,235 & 15,669 & 14,658 & 12,693 & 13,912 & 14,068 & 16,823 & 16,404 & 15,926 & 15,942 & 16,886 & 16,764 \\
\hline $10: 00$ p.m. & 16,094 & 15,462 & 14,565 & 12,647 & 13,864 & 14,019 & 16,881 & 16,447 & 15,963 & 16,001 & 16,738 & 15,614 \\
\hline 11:00 p.m. & 15,952 & 15,355 & 14,471 & 12,502 & 13,816 & 13,969 & 16,938 & 16,490 & 15,999 & 16,060 & 16,590 & 15,463 \\
\hline 12:00 Midnight & 15,811 & 15,248 & 14,378 & 12,457 & 13,768 & 13,919 & 16,821 & 16,402 & 15,926 & 15,944 & 16,442 & 15,313 \\
\hline \multicolumn{13}{|l|}{ Storage (acre-feet) } \\
\hline Maximum & 16,376 & 15,783 & 14,939 & 12,774 & 14,103 & 14,267 & 16,938 & 16,490 & 15,999 & 16,060 & 17,175 & 15,959 \\
\hline Minimum & 14,134 & 13,886 & 13,226 & 11,779 & 13,096 & 13,127 & 16,013 & 15,800 & 15,416 & 15,114 & 14,854 & 13,657 \\
\hline \multicolumn{13}{|l|}{ Elevation ( $f t)$} \\
\hline Maximum & $6,750.91$ & $6,748.84$ & $6,745.83$ & $6,737.70$ & $6,742.76$ & $6,743.37$ & $6,752.83$ & $6,751.30$ & $6,749.60$ & $6,749.81$ & $6,753.63$ & $6,749.46$ \\
\hline
\end{tabular}




\section{REFEREINCES}

Barnes, H.H., Jr. 1967, Roughness Characteristics of Natural Channels, U.S. Geological Survey Water-Supply Paper 1849.

Bureau of Reclamation, 1971, Designers' Opercting Criteria: Morrow Point Dam and Powerplant, Denver, Colo.

Chow, V.T., 1959, Open-Channel Hydraulics, McGraw-Hill, New York, N.Y.

Harris, R.E., 1992, "Gunnison River Study Flows - Aspinall Section 7 Consultation," memorandum from the Field Supervisor (U.S. Fish and Wildlife Service, Colorado/Utah Fish and Wildlife Enhancement, Salt Lake City, Utah) to Project Manager (Bureau of Reclamation, Grand Junction Project Office, Grand Junction, Colorado), April 16.

U.S. Army Corps of Engineers, 1982, HEC-2 Water Surface Profiles Users Manual, Hydrologic Engineering Center, Davis, Calif. 